\title{
Configurational force on a dynamic dislocation with localized oscillation
}

\author{
Soon Kim, Hokun Kim, Sung Youb Kim \\ Department of Mechanical Engineering, Ulsan National Institute of Science and Technology, Ulsan, 44919, South Korea
}

\section{A B S T R A C T}

Upon employing the conservation theorem and continuum theory, the configurational force on a singularity, or a defect, is given by a pathindependent integral called the $J$ integral. According to the continuum elasticity theory, the $J$ integral around a steadily moving dislocation is equal to the Peach-Koehler force acting on the dislocation and is independent of the integration path. However, using a discrete lattice dynamics method, we theoretically prove that the $J$ integral is not path-independent in practice even under uniform motion. This is because of the generation of phonons during the dislocation motion. In general, phonons are generated upon localized oscillation of the dislocation, and they dissipate energy from the dislocation core; consequently, a drag force is produced. As the drag force disturbs the dislocation motion, the $J$ integral around the moving dislocation is smaller than that around a stationary one, and its deviation from the stationary one corresponds to the drag force. In this study, we analytically derive the drag force for each oscillation mode by adopting dislocation-phonon coordinates. We classify the oscillation mode simply as symmetric or anti-symmetric after assuming the dislocation to be a localized defect having a finite core width. Consequently, the drag force is numerically calculated upon consideration of the discrete nature of the dislocation core. In particular, our study reveals that the anti-symmetric oscillation mode mainly contributes to the drag force in the limit of high dislocation velocity. Furthermore, we show that the resulting relation between the drag force and dislocation frequency can reproduce the dislocation velocity-stress curve. This work is expected to contribute to mesoand macro-scale plasticity when the material is loaded under extreme conditions or transient dislocation motion can be assumed.

\section{Introduction}

Macroscopic plastic deformation is an irreversible consequence of dislocation motion under external stress at the micro- and nanoscales. Therefore, numerous studies have been conducted to understand the plastic deformation in crystals, and accordingly, the collective dynamics of dislocation segments has been actively studied (Leung et al., 2015; Luscher et al., 2016, 2018). Especially, the more studies have been carried out to reflect the dislocation core effect on dislocation dynamics and plasticity in multi-scale framework (Amodeo et al., 2016; Liu et al., 2016; Jamond et al., 2016; Song et al., 2018; Swinburne et al., 2013; Zhang and Ngan, 2018). Although it has been well proved that the dislocation core determines the dislocation mobility and cross-slip rate, there has been lack of effort to include the core effect in dislocation density-based model. To overcome this limit, it is necessary to quantitatively describe the short-range interactions and discrete description of the dislocation core. In particular, the dislocation core effect becomes more significant when the material is under dynamic or shock loadings (Gurrutxaga-Lerma et al., 2015; Shehadeh and Zbib, 2016; Cui et al., 2019) because the dislocation inertia term (, or effective mass), which is responsible to energy radiation from the accelerated dislocation, is not negligible. Recently, Gurrutxaga-Lerma et al. (2015) revealed that the dynamic yield stress is determined by the interference between elastic waves emitted from the dislocation core and elastic shockwave generated by rapid compression of the material.

\footnotetext{
* Corresponding author. EB5 801-6, 50 UNIST-gil, UNIST, Ulsan, 44919, South Korea.

E-mail address: sykim@unist.ac.kr (S.Y. Kim).
} 
The dislocation core effect can be quantified by solving an equation of its motion. For this, the local driving force acting on the dislocation must be quantified. However, unlike the Newtonian force, the mass is not defined for defects (including the dislocation). Instead, the net translational force on the defect can be quantified by defining a configurational force, or the $J$ integral, given in the form of an integral (Eshelby, 1951; Rice, 1968). Without defining the defect mass, the $J$ integral is directly obtained using the derivative of the Lagrangian of the system with respect to the translation coordinate of the defect. The $J$ integral physically indicates the energy release rate for the defect translation, and the conservation theorem was used to prove that the $J$ integral is independent of the integration path around a stationary defect (Noether, 1971). Therefore, the $J$ integral has been widely used to predict the start and the direction of crack propagation (Kaczmarczyk et al., 2014; Özenç et al., 2016; Guo and Li, 2017), effective fracture toughness of heterogeneous media (Hossain et al., 2014; Kuhn and Müller, 2016) and damage evolution inside the materials (Yuan and Li, 2019). When the $J$ integral is applied to the stationary dislocation, the $J$ integral is equal to the Peach-Koehler (PK) force acting on it (Eshelby, 1956). The $J$ integral has been widely applied to characterize various states of dislocations upon consideration of simple to complex cases (Lubarda and Markenscoff, 2007; Baxevanakis and Giannakopoulos, 2015; Ballarini and Royer-Carfagni, 2016; Lubarda, 2015; Seo et al., 2018).

The $J$ integral has been applied to dynamic defects, as well as stationary ones (Ni and Markenscoff, 2008; Arvanitakis et al., 2012; Kuhn and Müller, 2016; Özenç et al., 2016). Compared with the stationary $J$ integral, the pseudo-momentum term additionally contributes to the dynamic $J$ integral, but the path-independency is still maintained as long as the defect moves with a uniform velocity (Ni and Markenscoff, 2008). Consequently, the dynamic $J$ integral is equal to the PK force acting on the dislocation, as in steady motion. Nevertheless, it is worthwhile to mention the drag force $\mathbf{F}^{\text {drag }}$ acting opposite to the local driving force during the dislocation motion because it always exists as long as the dislocation glides. As the drag force is attributed to the rate of energy dissipation $\dot{Q}$ from the dislocation core, these terms can be related by $\dot{Q}=-\mathbf{F}^{\text {drag }} \cdot \mathbf{v}$, where $\mathbf{v}$ is the dislocation velocity. Moreover, as the drag force influences the dislocation inertia through the equation of dislocation motion, the dislocation core width and its self-force change over time when the dislocation moves non-uniformly (Pellegrini, 2012). This implies that the drag may have influence on the configurational force on the moving dislocation.

The energy dissipation mechanisms caused by the drag force depend on external conditions. At a very low temperature (where the thermal effect is ignorable), the energy dissipation during the dislocation motion mainly originates from radiation damping. The radiation damping is generated by the nonlinearity-induced energy loss from long-to short-wavelength phonons (Kresse and Truskinovsky, 2003). By assuming the dislocation as an instability moving with uniform velocity in a discrete lattice, the rate of dissipation and the configurational force can be analytically derived as functions of the dislocation velocity (Wang and Abeyaratne, 2018). The discrete lattice model predicts that more phonons are emitted, and the emitted phonons have shorter wavelengths, as the dislocation velocity decreases. Furthermore, the emitted phonons are scattered by an anharmonic strain field around the dislocation core, which reduces the average stress around the dislocation core (Kim et al., 2016). If the thermal effect is considered, additional drag mechanisms appear during the dislocation motion. In general, the influence of temperature on the drag mechanisms has been examined by normalizing it with respect to the Debye temperature, $\theta_{D}$. (In most crystals, $\theta_{D}$ is approximately $200-400 \mathrm{~K}$.) If the temperature is lower than approximately $0.1 \theta_{D}$, phonon emission owing to the vibrating dislocation in the thermal field becomes the predominant source of energy dissipation (Ninomiya, 1974; Chen et al., 2017; Amrit et al., 2018). This is called flutter drag. In contrast to radiation damping, where only the excited waves whose phase velocities are equal to the dislocation velocity are radiated, arbitrary waves are emitted by the flutter drag. Furthermore, at a temperature higher than or equal to $\theta_{D}$, phonon wind becomes the predominant drag mechanism (Al'Shitz and Indenbom, 1975; Blaschke, 2019a, 2019b; Blaschke et al., 2020a). In this case, anharmonic scattering of phonons by the moving dislocation imparts momentum to the latter in the opposite direction to the dislocation motion. In particular, when the dislocation velocity is comparable to the transverse sound wave speed, the aforementioned drag forces are amplified owing to the relativistic effect according to the continuum elasticity theory. Moreover, it has recently been proven that the relativistic effect can be alternatively explained by characterizing the dislocation as a spontaneous vibrating defect based on the discrete lattice dynamics (Kim et al., 2020).

In this study, we theoretically derive the $J$ integral around a uniformly moving dislocation by considering the drag effect. We characterize the drag effect by assuming that the dislocation oscillates based on the lattice dynamics theory. Unlike the continuum theory, the lattice dynamics theory assumes the frequency spectrum of the system to be bounded. Therefore, the divergence problems that are presented in the field theory can be avoided. Depending on whether the frequencies newly generated by the dislocation deviate from the frequency band of the perfect lattice, the oscillation types are classified into two cases. First, if the dislocation frequency is above the maximum frequency of a perfect lattice, the oscillation amplitude is localized around the dislocation core; hence, it exponentially decays as the distance from the core increases. Thus, the dislocation core can be treated as an isolated defect (Lifshitz and Kosevich, 1966). This type of oscillation can be applied to a dynamic dislocation when the phonons are localized around the dislocation. In contrast, if the dislocation frequency exists within the frequency band of a perfect lattice, the waves are scattered rather than being localized. Thus, this type of oscillation can be applied when the phonon scattering is the dominant drag mechanism. Here, we only deal with the former type of oscillation, or the localized oscillation. And we express the drag force by using the dislocation-phonon coordinates rather than conventional atomic displacement coordinates. Subsequently, the dislocation oscillation is examined by solving a matrix eigenvalue problem, and finally, the contribution of each eigenmode to the drag force is revealed by considering the discrete nature of the dislocation core. 


\section{Continuum approach (revisited)}

Before we discuss the main results of our study in the next section, we briefly introduce a derivation of the configurational force acting on a uniformly moving dislocation based on the continuum theory. This derivation was undertaken by Stroh (1962). We summarize it here to show that the $J$ integral calculated for a static dislocation can still be identically applied to a moving dislocation, unless it accelerates. The detailed derivation is presented in Appendices A and B based on Stroh's (1962) work.

Imagine a system consisting of two separated regions I and II as in Fig. 1. In region I, a dislocation loop, which is denoted as $D$, is inserted and it is surrounded by a surface $\Sigma_{i}$. Thus, $\Sigma_{i}$ has a tube shape whose radius is $a$. Assume that there are other singularities or stress sources, denoted as $S$, in region II, which are surrounded by the inner and outer surfaces, $\Sigma_{i}$ and $\Sigma_{o}$, respectively. Thus, we can express the total Lagrangian of the system, $L$, as

$$
L=L^{D}+L^{S}+L^{D S}
$$

where $L^{D}$ and $L^{S}$ are the Lagrangians of $D$ and $S$, respectively, and $L^{D S}$ is the Lagrangian caused by the interaction between them. As the dislocation moves, the distance between $D$ and $S$ changes, which will cause a change in $L^{D S}$. If $\xi$ specifies the position of the dislocation, the configurational force acting on the dislocation along the $\xi$ direction, $F_{\xi}$, is defined as

$$
\int_{t_{1}}^{t_{2}} F_{\xi} \delta \xi d t=\delta \int_{t_{1}}^{t_{2}} L^{D S} d t
$$

According to Appendix A, $L^{D S}$ is derived as

$$
L^{D S}=T^{D S}-U^{D S}=-b_{i} \int_{C} \sigma_{i j}^{S} d S_{j}+\int_{I+I I} \rho \frac{d}{d t}\left(u_{i}^{D} v_{i}^{S}\right) d^{3} x
$$

where $\sigma^{S}$ and $v^{S}$ are the stress and material velocity caused by $S$, respectively, and $u^{D}$ is the displacement caused by $D$. Here, $\rho$ is the density of the system. The configurational force can be derived by using Eq. (2) and Eq. (3). Assume that the dislocation loop, $D$, moves by an infinitesimal distance, $\delta \xi$, as shown in Fig. 2. Then, according to Appendix B, a variation of Eq. (3) can be derived as

$$
\delta L^{D S}=\delta L_{1}^{D S}+\delta L_{2}^{D S}=b_{i} \oint \sigma_{i j}^{S} \varepsilon_{j k l} d s_{k} \delta \xi_{l}+\int_{I+I I} \rho \frac{d}{d t}\left(\delta u_{i}^{D} v_{i}^{S}\right) d^{3} x
$$

where

$$
\begin{aligned}
& \delta L_{1}^{D S}=b_{i} \oint \sigma_{i j}^{S} \varepsilon_{j k l} d s_{k} \delta \xi_{l} \\
& \delta L_{2}^{D S}=\int_{I+I I} \rho \frac{d}{d t}\left(\delta u_{i}^{D} v_{i}^{S}\right) d^{3} x .
\end{aligned}
$$

Then, the force acting on $D$, to which $\delta L_{1}^{D S}$ contributes, is derived as Eq. (7), which is the PK force, from Eq. (2).

$$
F_{\xi}^{1}=b_{i} \sigma_{i j}^{S} \varepsilon_{j k l} t_{k},
$$

where $t_{k}$ is the unit dislocation line vector. Furthermore, $\delta L_{2}^{D S}$ is derived as

(a)

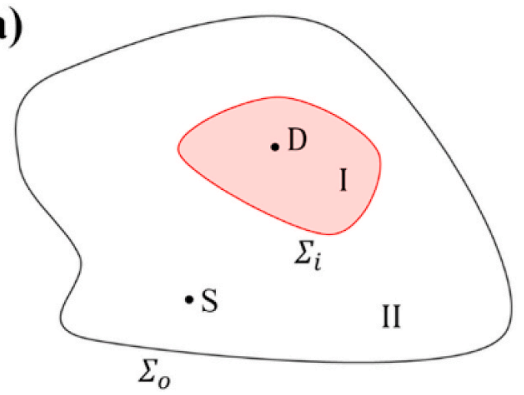

(b)

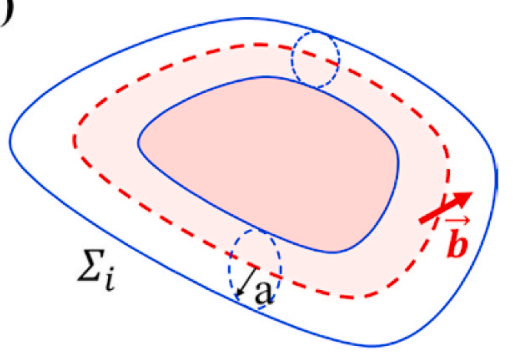

Fig. 1. (a) System including a dislocation loop $D$ in region I and other singularities $S$ in region II. (b) Enlarged view of the dislocation loop $D$. Here, the dislocation is surrounded by a cylindrical tube whose radius is $a$. In both (a) and (b), the shaded region represents the area slipped by the dislocation loop. 


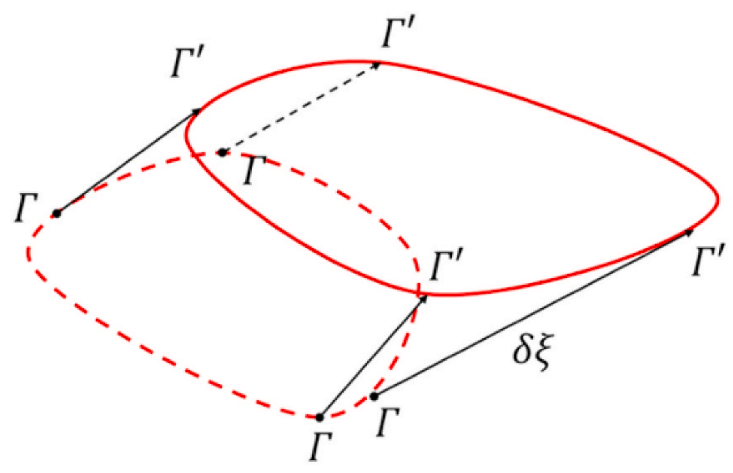

Fig. 2. Motion of the dislocation loop.

$$
\delta L_{2}^{D S}=\int_{\Sigma_{i}} \rho \delta u_{i}^{D} v_{i}^{S} \dot{\xi}_{j} d S_{j}+\int_{I} \rho v_{i}^{S} \frac{d}{d t}\left(\delta u_{i}^{D}\right) d^{3} x .
$$

Here, $\delta L_{2}^{D S}$ depends on the material velocity, $v^{S}$, or the velocity of the observer. In this study, the frame of the observer does not move so that $v^{S}=0$. Therefore, the configurational force acting on the dynamic dislocation is calculated by using Eq. (7), which is equal to the PK force defined for a stationary dislocation. However, even if the frame of the observer moves, the equivalence is still satisfied, unless the frame accelerates. This is because we can make $\delta u^{D}$ arbitrarily small inside region I, according to the continuity assumption, by suitably restricting $\delta \xi$. Consequently, it is possible to make the contribution of $\delta L_{2}^{D S}$ arbitrarily small compared with that of $\delta L_{1}^{D S}$ to the configurational force. In fact, Eq. (8) is responsible for a force called the Lorentz force, but it was proved that this force does not influence the dislocation glide (Lothe, 1961; Stroh, 1962; Kosevich, 1965).

\section{Discrete lattice approach}

\subsection{Coordinate transformation}

In mechanics, the Lagrangian of a system is generally described in terms of the displacements of constituent atoms. However, as we intend to describe the interaction between the strain field around the dislocation and the phonons directly, we change the conventional displacement coordinate system to a dislocation-phonon coordinate system by adopting the dislocation coordinate, which was first suggested by Ninomiya (1972). First, we introduce the coordinate transformation based on Ninomiya's (1972) work. Subsequently, we define the $J$ integral around a dynamic dislocation in the transformed coordinate system.

We simplify the system into a one-dimensional chain consisting of $N$ atoms. Thus, the system has $N$ degrees of freedom (DOFs). However, as a single dislocation is inserted inside the system, the DOFs of the system are reduced to $N-1$, as 1 -DOF is assigned to

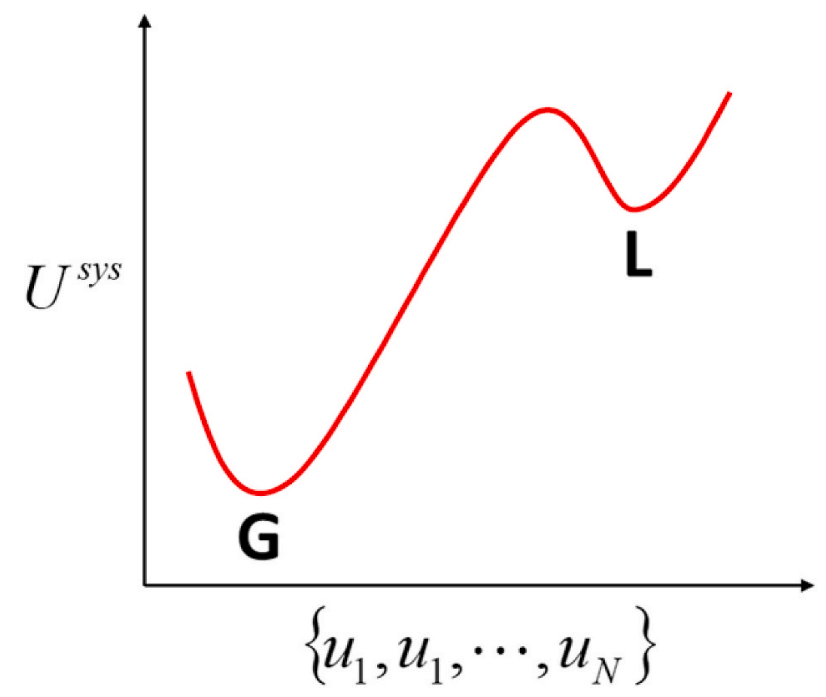

Fig. 3. Simple description of the $N$-dimensional potential energy surface as a function of atomic displacements. If there is no defect, the system is located at a valley "G." In contrast, if the dislocation is inserted, then the system is located at a valley "L." 
describe the strain field of the dislocation. If we plot the energy of the system as a function of $N$ dimensional abscissas, $\left\{u_{1}, u_{2}, \cdots, u_{N}\right\}$, where $u_{n}$ corresponds to the displacement of the $n$-th atom, the energy surface is presented as shown in Fig. 3 . The figure shows two valleys. The global minimum state (state G) corresponds to the system with no defects, whereas the local minimum state (state L) corresponds to the system where the dislocation is inserted. As the dislocation glides, the state of the system will move along the local minimum valley on the $N$-dimensional energy surface with the change in the displacement of each atom over time. Accordingly, we can define the dislocation coordinate, $\xi$, as a collective variable expressed in terms of $\left\{u_{1}^{\text {dis }}, u_{2}^{\text {dis }}, \cdots, u_{N}^{\text {dis }}\right\}$, where $u_{n}^{\text {dis }}$ is the displacement of the $n$-th atom required to accommodate the dislocation. Physically, $\xi$ represents the position of the dislocation. If 1 -DOF is assigned to $\xi$, then $N-1$ DOFs remain. These remaining DOFs are orthogonal to $\xi$, and hence, they induce the climbing of the sides of the local minimum valley in Fig. 3. Physically, these $N-1$ variables correspond to the phonons that disturb the dislocation glide along the local energy minimum valley, and hereafter, we define them as phonon coordinates.

If the dislocation is in a static equilibrium state, the displacement field around it, $w^{s}(x-\xi)$, can be described as shown in Fig. 4(a). If it is defined by using $\lambda^{(1)}(x-\xi)$,

$$
w^{s}(x-\xi)=\int_{-\infty}^{\xi} \lambda^{(1)}\left(x-\xi^{\prime}\right) d \xi
$$

then $\lambda^{(1)}(x-\xi)=d w^{s} / d \xi$ is satisfied and it becomes a localized function around $\xi$ as described in Fig. 4(b). Here, $\lambda^{(1)}(x-\xi)$ is normalized as

$$
\sum_{n}\left(\lambda^{(1)}(n b-\xi)\right)^{2}=1
$$

Moreover, $w^{s}(x-\xi)$ should satisfy Eq. (11) as a boundary condition.

$$
\lim _{\xi \rightarrow \infty} w^{s}(x-\xi)=b
$$

where $b$ is the Burgers vector. Hereafter, we use the alternate expression $\lambda_{n}^{(1)}(\xi) \equiv \lambda^{(1)}(n b-\xi)$ and $w_{n}(\xi) \equiv w^{s}(n b-\xi)$ for convenience. From the shape of $\lambda_{n}^{(1)}(\xi)$ in Fig. 4(b), it can be inferred that $\lambda_{n}^{(1)}(\xi)$ physically indicates the compactness of the dislocation core.

Moreover, if we define additional variables, $\lambda_{n}^{(j)}(\xi)$ for $j=1,2, \cdots, N$ and express the atomic displacement $u_{n}$ by using $\left\{\lambda_{n}^{(1)}, \lambda_{n}^{(2)}, \cdots\right.$, $\left.\lambda_{n}^{(N)}\right\}$, Eq. (12) can be derived.

$$
u_{n}=w_{n}(\xi)+\sum_{v=2}^{N} \tau_{v} \lambda_{n}^{(v)}(\xi)
$$

Here, as $\lambda_{n}^{(1)}, \lambda_{n}^{(2)}, \cdots, \lambda_{n}^{(N)}$ should be orthogonal to each other, Eq. (13) follows.

$$
\sum_{n} \lambda_{n}^{(\mu)}(\xi) \lambda_{n}^{(v)}(\xi)=\delta_{\mu v}(\mu, v=1,2, \cdots, N),
$$

where $\delta_{\mu v}$ is the Kronecker delta. As the dislocation glides, phonons are generated, and they cause an additional atomic displacement, which makes the system deviate from the static equilibrium state. The contribution of the phonons to the displacement is reflected by the second term on the right-hand side (RHS) of Eq. (12). By using Eq. (12), the Lagrangian can be expressed in terms of the new coordinates $\left\{\xi, \tau_{2}, \cdots, \tau_{N}\right\}$. However, it is not practical because $\lambda_{n}^{(2)}, \cdots, \lambda_{n}^{(N)}$ are not known and the constraint, Eq. (13), complicates the calculation.

To avoid these drawbacks, Ninomiya (1972) suggested a method in which a basis set is changed by adding a single redundant variable, $\tau_{1}$, to the system. Consequently, the total number of variables becomes $N+1$ and the DOF of the system becomes $N$. Physically, the addition of the variable corresponds to the addition of an isolated free oscillator to the dislocated system, $L^{\text {sys }}$. The
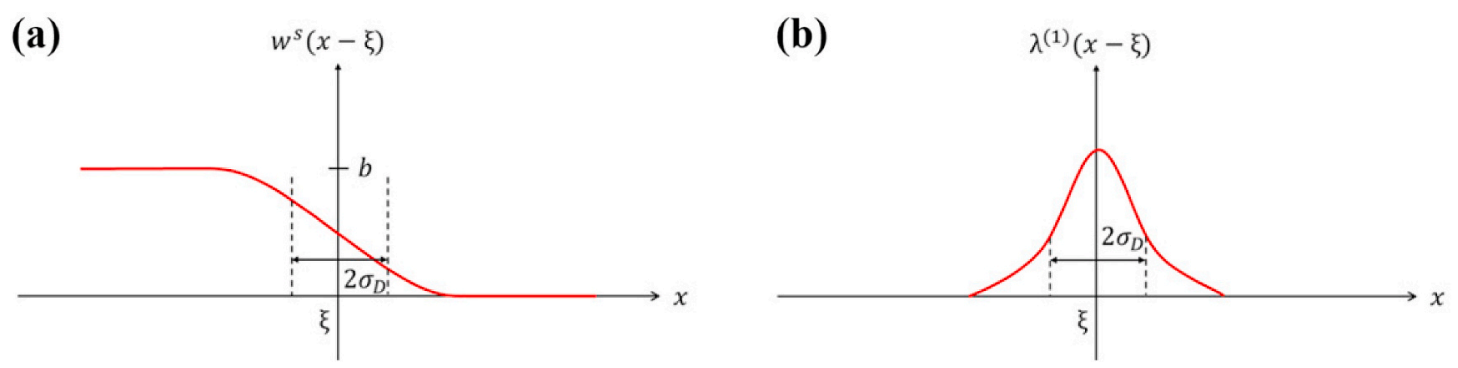

Fig. 4. (a) Dislocation displacement field under a static equilibrium state. (b) Compactness function of the dislocation core. Here, $\lambda^{(1)}(x-\xi)=d w^{s} /$ $d \xi$ and $\lambda^{(1)}$ is assumed as a Gaussian distribution function whose mean is $\xi$ and standard deviation is $\sigma_{D}$. We assumed that the dislocation core width is $2 \sigma_{D}$ in this study. 
Lagrangian of the added oscillator is defined as

$$
L_{\text {add }}=\frac{1}{2} m \dot{\tau}_{1}^{2}-\frac{1}{2} m \omega_{1}^{2} \tau_{1}^{2}
$$

where $m$ is the atomic mass and $\omega_{1}$ is the frequency of the added oscillator. And if we define new variables as

$$
\begin{aligned}
& v_{n}=\sum_{v=1}^{N} \tau_{v} \lambda_{n}^{(v)}(\xi), \\
& \tau_{v}=\sum_{n=1}^{N} v_{n} \lambda_{n}^{(v)}(\xi),
\end{aligned}
$$

we can express $u_{n}$ and $\tau_{1}$, respectively, in terms of these new variables as

$$
\begin{aligned}
& u_{n}=w_{n}(\xi)+\sum_{v=2}^{N} \tau_{v} \lambda_{n}^{(v)}(\xi)=w_{n}(\xi)+v_{n}-\tau_{1} \lambda_{n}^{(1)}(\xi)=w_{n}(\xi)+v_{n}-\left(\sum_{m=1}^{N} v_{m} \lambda_{m}^{(1)}(\xi)\right) \lambda_{n}^{(1)}(\xi) \\
& \tau_{1}=\sum_{n=1}^{N} v_{n} \lambda_{n}^{(1)}(\xi) .
\end{aligned}
$$

In Eq. (16-1) and Eq. (16-2), as $\lambda_{n}^{(2)}, \cdots, \lambda_{n}^{(N)}$ are not required, whereas only $\lambda_{n}^{(1)}$, which is explicitly known, is used, the coordinate transformation from $\left\{u_{1}, u_{2}, \cdots, u_{N}, \tau_{1}\right\}$ to $\left\{\xi, v_{1}, v_{2}, \cdots, v_{N}\right\}$ can be conveniently undertaken. Furthermore, the orthogonality constraint is automatically reflected to relate $v_{n}$ and $\tau_{v}$ in Eq. (15), and no constraint is necessary. Consequently, the transformed coordinate system consists of the dislocation coordinate, $\xi$, and $N$ phonon coordinates, $v_{1}, v_{2}, \cdots, v_{N}$.

\section{2. $J$ integral in dislocation-phonon coordinate system}

The $J$ integral can be derived by using the transformed coordinate system, $\left\{\xi, v_{1}, v_{2}, \cdots, v_{N}\right\}$. As the $J$ integral physically indicates a translational configurational force per unit length of the dislocation, it can be defined by using the dislocation coordinate as

$$
J=-\frac{1}{l} \frac{\partial L^{s y s}}{\partial \xi}=-\frac{1}{l}\left(\frac{\partial T^{s y s}}{\partial \xi}-\frac{1}{l} \frac{\partial U^{s y s}}{\partial \xi}\right)
$$

where $l$ is the length of the dislocation, and $T^{\text {sys }}$ and $U^{\text {sys }}$ are the kinetic and potential energies of the system, respectively. Here, $T^{\text {sys }}$ and $U^{\text {sys }}$ are expressed, respectively, as

$$
\begin{aligned}
T^{s y s} & =\frac{1}{2} m \sum_{n=1}^{N} \dot{u}_{n}^{2}, \\
U^{s y s} & =\frac{1}{2} \gamma \sum_{n=2}^{N}\left(u_{n}-u_{n-1}\right)^{2}+\sum_{n=1}^{N} V\left(u_{n}\right),
\end{aligned}
$$

where $\gamma$ is the spring constant and $V$ is the substrate potential. The kinetic term in Eq. (17) becomes zero and this is proved in Appendix C. Similarly, the potential term in Eq. (17) is expressed as follows:

$$
\frac{\partial U^{s y s}}{\partial \xi}=\sum_{n=1}^{N} \frac{\partial U^{s y s}}{\partial u_{n}} \frac{\partial u_{n}}{\partial \xi}+\frac{\partial U^{s y s}}{\partial \tau_{1}} \frac{\partial \tau_{1}}{\partial \xi}
$$

From Eq. (16-1),

$$
\frac{\partial u_{n}}{\partial \xi}=\frac{d w_{n}}{d \xi}-\left[\frac{d \lambda_{n}^{(1)}}{d \xi} \sum_{m=1}^{N} \lambda_{m}^{(1)} v_{m}+\lambda_{n}^{(1)} \sum_{m=1}^{N} \frac{d \lambda_{m}^{(1)}}{d \xi} v_{m}\right]
$$

is derived. By using $\partial U^{\text {sys }} / \partial \tau_{1}=0$, and substituting Eq. (19) andEq. (20) into Eq. (17), the $J$ integral is derived as

$$
J=\frac{1}{l} \sum_{n=1}^{N} \frac{\partial U^{s y s}}{\partial u_{n}} \frac{d w_{n}}{d \xi}-\frac{1}{l} \sum_{n=1}^{N} \frac{\partial U^{s y s}}{\partial u_{n}}\left(\frac{d \lambda_{n}}{d \xi} \sum_{m=1}^{N} \lambda_{m} v_{m}+\lambda_{n} \sum_{m=1}^{N} \frac{d \lambda_{m}}{d \xi} v_{m}\right)
$$

Here, $\lambda_{n}^{(1)}$ was replaced by $\lambda_{n}$ for convenience.

Given that $\lambda_{n}(\xi)$ is a localized function around $\xi$, we assume that it is a Gaussian distribution function as defined in Eq. (22).

$$
\lambda_{n}(\xi)=\lambda(r ; \xi)=\frac{b}{\sigma_{D} \sqrt{2 \pi}} e^{-\frac{(r-\xi)^{2}}{2 \sigma_{D}^{2}}}
$$


where $r=n b$ and $\sigma_{D}$ is the standard deviation of the distribution function and is a parameter characterizing the size of the dislocation core. In other words, we describe the dislocation core as a diffusive zone, whose width is approximately $2 \sigma_{D}$. Note that Eq. (22) also satisfies the boundary condition, Eq. (11). As $\partial U^{s y s} / \partial u_{n}=\left(\tau_{a p p}+\tau_{d i s}\right) l b$, where $\tau_{d i s}$ is the dislocation stress field and $d w_{n} / d \xi=\lambda_{n}$, the first term on the RHS of Eq. (21) in the continuum limit becomes

$$
J_{1}=\frac{1}{l} \sum_{n} \frac{\partial U^{s y s}}{\partial u_{n}} \frac{d w_{n}}{d \xi} \approx \frac{1}{l b} \int_{-\infty}^{\infty} \frac{\partial U^{s y s}}{\partial u} \frac{d w}{d \xi} d r=\int_{-\infty}^{\infty}\left(\tau_{a p p}+\tau_{d i s}\right) \lambda(r ; \xi) d r .
$$

According to the linear elasticity theory, as $\tau_{\text {dis }} \sim 1 / r, \tau_{\text {dis }}=C / r$, where $C$ is a constant, is substituted into Eq. (23). Moreover, we assume that the dislocation is at the origin, and hence, $\xi=0$. Subsequently, Eq. (23) results in Eq. (24). Here, the $\tau_{d i s}$ term disappears because it is an odd function.

$$
J_{1} \approx \frac{b}{\sigma_{D} \sqrt{2 \pi}} \int_{-\infty}^{\infty}\left(\tau_{a p p}+\frac{C}{r}\right) e^{-\frac{r^{2}}{2 \sigma_{D}^{2}}} d r=\tau_{a p p} b .
$$

Therefore, the first term of Eq. (21) is equal to the PK force, $F_{P K}$, defined in the continuum theory. Unlike the first term, the second term of Eq. (21) includes the phonon coordinates, $v_{n}$. Accordingly, it can be inferred that the second term in Eq. (21) is generated because of the interaction between the dislocation and the phonons. This term is the main interest of this study, and we define it as $F_{d r a g}$. Consequently, the $J$ integral around the dynamic dislocation that interacts with the phonons consists of two separate terms, i.e.,

$$
J=F_{P K}+F_{\text {drag }}
$$

where

$$
\begin{aligned}
& F_{P K}=\tau_{a p p} b \\
& F_{\text {drag }}=-\frac{1}{l} \sum_{n=1}^{N} \frac{\partial U^{s y s}}{\partial u_{n}}\left(\frac{d \lambda_{n}}{d \xi} \sum_{m=1}^{N} \lambda_{m} v_{m}+\lambda_{n} \sum_{m=1}^{N} \frac{d \lambda_{m}}{d \xi} v_{m}\right) .
\end{aligned}
$$

As an extreme example, if the dislocation does not move, phonons are not generated from the dislocation core. Consequently, $v_{n}$ will be zero for every atom; hence, the $J$ integral will be equal to $F_{P K}$, as in the continuum theory. Thus, Eq. (25) reconfirms that the phonons are responsible for dragging the moving defect (Slepyan, 2001; Kresse and Truskinovsky, 2003; Kim et al., 2016).

To simplify Eq. (26-2), $C$ in $\tau_{\text {dis }}$ should be analytically obtained using the following process. As it was proved that Eq. (23) is equal to the PK force in the continuum limit, the $M$ integral can be defined in the discrete lattice similarly. Based on the definition of the $M$ integral in the continuum theory (Budiansky and Rice, 1973; Agiasofitou and Lazar, 2017), $M_{1}$ with no drag effect can be simply derived in the continuum limit as

$$
M_{1} \approx \int_{-\infty}^{\infty} r\left(\tau_{\text {app }}+\tau_{d i s}\right) \lambda(r ; \xi) d r=\int_{-\infty}^{\infty} r\left(\tau_{a p p}+\frac{C}{r}\right) \lambda(r ; \xi) d r=C b .
$$

Here, the $\tau_{a p p}$ term has disappeared, as $r \lambda(r)$ is an odd function. According to the continuum theory, $M_{1}=G b^{2} / 4 \pi$ in a twodimensional system. Here, $G$ is shear modulus. Therefore, $C=G b / 4 \pi$ is obtained. Then, as we replace the discrete sums with integrals from $r=-R$ to $r=R$ in Eq. (26-2), $F_{\text {drag }}$ becomes

$$
\begin{aligned}
& -F_{\text {drag }}=\frac{1}{l} \sum_{n=1}^{N} \frac{\partial U^{\text {sys }}}{\partial u_{n}}\left(\frac{d \lambda_{n}}{d \xi} \sum_{m=1}^{N} \lambda_{m} v_{m}+\lambda_{n} \sum_{m=1}^{N} \frac{d \lambda_{m}}{d \xi} v_{m}\right) \\
& =\frac{1}{l}\left[\left(\sum_{n} \frac{\partial U^{s y s}}{\partial u_{n}} \frac{d \lambda_{n}}{d \xi}\right)\left(\sum_{m=1}^{N} \lambda_{m} v_{m}\right)+\left(\sum_{n} \frac{\partial U^{s y s}}{\partial u_{n}} \lambda_{n}\right)\left(\sum_{m=1}^{N} \frac{d \lambda_{m}}{d \xi} v_{m}\right)\right] \\
& =\left[\int_{-R}^{R}\left(\tau_{a p p}+\frac{G b}{4 \pi r}\right) \frac{d \lambda}{d \xi} d r \cdot \frac{1}{b} \int_{-R}^{R} \lambda(r) v(r) d r\right]+\left[\int_{-R}^{R}\left(\tau_{a p p}+\frac{G b}{4 \pi r}\right) \lambda(r) d r \cdot \frac{1}{b} \int_{-R}^{R} \frac{d \lambda}{d \xi} v(r) d r\right] .
\end{aligned}
$$

If we substitute $d \lambda / d \xi=\left[b(r-\xi) /\left(\sigma_{D}^{3} \sqrt{2 \pi}\right)\right] \exp \left(-(r-\xi)^{2} / 2 \sigma_{D}^{2}\right)$ with $\xi=0$ into Eq. (28) and delete the odd terms, Eq. (28) is simplified to

$$
F_{\text {drag }}=-\frac{b}{\sigma_{D}^{3} \sqrt{2 \pi}}\left[\frac{G b}{4 \pi} \int_{-R}^{R} v(r) e^{-\frac{r^{2}}{2 \sigma_{D}^{2}}} d r+\tau_{a p p} \int_{-R}^{R} r v(r) e^{-\frac{r^{2}}{2 \sigma_{D}^{2}}} d r\right] .
$$

If we restore the discreteness, then the drag force can also be represented as 


$$
F_{\text {drag }}=-\frac{b}{\sigma_{D}^{3} \sqrt{2 \pi}}\left[\frac{G b^{2}}{4 \pi} \sum_{j=-R / b}^{R / b} v(j) e^{-\frac{(j b)^{2}}{2 \sigma_{D}^{2}}}+\tau_{a p p} b^{2} \sum_{j=-R / b}^{R / b} j v(j) e^{-\frac{(j b)^{2}}{2 \sigma_{D}^{2}}}\right] .
$$

This will be used for the numerical calculation of the drag force in Section 4. Two points can be inferred from the closed form of Eq. (29). First, the phonons contribute to the drag force in two ways by interacting with the strain fields caused by the dislocation and external stress. Among them, the interactions near the dislocation core are mainly responsible for the drag force because the exponential terms in Eq. (29) decay rapidly, as the distance from the center of the core increases. Second, the drag force depends on the integration size, $R$. In other words, the existence of the drag force makes the $J$ integral path-dependent.

\subsection{Phonon coordinates and eigenmodes}

According to Eq. (29) (or Eq. (30)), the drag force can be calculated only after the phonon coordinates, $v(r)$ (or $v(j)$ ), are determined. In general, a wave can be analyzed by decomposing it into elementary normal modes (called eigenmodes) depending on the DOF of the system. Similarly, the phonon coordinate, or the oscillation displacement, can be decomposed into a finite number of eigenmodes, where all the atoms oscillate with the same frequency. Given that the phonon coordinate is expressed by a linear combination of the eigenmodes, determining the contribution of each eigenmode to the drag force is our primary objective. In this section, we derive the phonon coordinate of each eigenmode by simplifying the dislocation as a localized defect based on the method developed by Montroll and Potts (1955) and investigate the influence of the phonon coordinate on the drag force. The authors analyzed the oscillation of a point defect by solving an eigenvalue problem. However, as the dislocation core width is an important factor to determine the drag force in this study, we extended their method to a defect whose core has a finite width.

In a one-dimensional atomic chain with a defect, the displacement of the $j$-th atom can be obtained by solving Eq. (31).

$$
m \ddot{x}_{j}+\gamma\left[x_{j+1}-2 x_{j}+x_{j-1}\right]=D x_{j},
$$

where $m$ is the atomic mass, $\gamma$ is the spring constant, and $D$ is an operator characterizing the defect or dislocation. Under the plane wave assumption, $x_{j}=v(j) \exp (-i \omega t)$, Eq. (31) is modified to

$$
L v(j)=m \omega^{2} v(j)+\gamma[v(j+1)-2 v(j)+v(j-1)]
$$

where $L=-D$. Furthermore, we can express the RHS of Eq. (32) in a matrix form by defining $w^{(i)}(j)$ as

$$
L v(j)=m \omega^{2} v(j)+\gamma[v(j+1)-2 v(j)+v(j-1)] \equiv \sum_{k} w^{(k)}(k+j) v(k+j) .
$$

Before solving Eq. (33), it is convenient to define a function $g(j)$ that satisfies

$$
L g(x-s)=\gamma \delta(x-s) \text {. }
$$

Here, $g(j)$ is the (lattice) Green's function. If we multiply an arbitrary function, $f(s)$, to both sides of Eq. (34) and integrate over $S$, then Eq. (35) is derived.

$$
f(x)=\frac{1}{\gamma} \int L g(x-s) f(s) d s .
$$

Therefore, to solve the equation $L v(j)=f(j)$, it can be alternatively expressed by

$$
L v(j)=\frac{1}{\gamma} \int L g(j-s) f(j) d s=L\left(\frac{1}{\gamma} \int g(j-s) f(j) d s\right) .
$$

Thus, we can obtain $v(j)$ as

$$
v(j)=\frac{1}{\gamma} \int g(j-s) f(j) d s .
$$

From Eq. (33) and Eq. (37),

$$
v(j)=\gamma^{-1} \sum_{m} \sum_{k} g(j-m) w^{(k)}(k+m) v(k+m) .
$$

If $k+m$ is replaced by $l$, then $v(j)$ is derived as

$$
v(j)=\gamma^{-1} \sum_{m} \sum_{l} g(j+k-l) w^{(k)}(l) v(l)
$$

To define $g(j)$, let us express it in terms of the Fourier transform as 


$$
g(j)=\frac{1}{2 \pi} \int_{-\infty}^{\infty} G(\varphi) e^{-i \varphi j} d \varphi .
$$

By substituting Eq. (40) into Eq. (32) and using Eq. (34), Eq. (41) is derived.

$$
\begin{aligned}
\gamma \delta(j) & =\frac{1}{2 \pi} \int_{-\infty}^{\infty} G(\varphi)\left[m \omega^{2}+2 \gamma(\cos \varphi-1)\right] e^{-i \varphi j} d \varphi . \\
\text { As } \delta(j) & =(1 / 2 \pi) \int_{-\infty}^{\infty} \exp (-i \phi j) d \phi \\
G(\varphi) & =\frac{\gamma}{m \omega^{2}+2 \gamma(\cos \varphi-1)} .
\end{aligned}
$$

If we substitute Eq. (42) into Eq. (40) and confine the integration range to the first Brillouin zone, the Green's function is derived as

$$
g(j)=\frac{\gamma}{\pi} \int_{0}^{\pi} \frac{\cos \varphi j}{m \omega^{2}+2 \gamma(\cos \varphi-1)} d \varphi .
$$

Depending on the frequency, $\omega$, the integrand of Eq. (43) either has poles or does not. In a perfect lattice system without any defects, the maximum reachable frequency, $\omega_{L}$, is $2 \sqrt{\gamma / m}$. If $\omega \leq \omega_{L}$, the integrand has poles, but if $\omega>\omega_{L}$, it does not have poles. In this study, we only consider the latter. In this case, the frequency bands newly generated by embedding the dislocation are localized around the atoms of the dislocation core (Kim et al., 2020). Therefore, $v(j) \rightarrow 0$ as $j \rightarrow \pm \infty$. In general, this occurs when the drag force is governed by the inertial oscillation resulting from the extremely high speed of the dislocation (Gurrutxaga-Lerma, 2016; Peng et al., 2019). If we define $f \equiv \omega / \omega_{L} \equiv \cosh (z / 2)$ and assume that $f>1$, then Eq. (43) is modified to

$$
g(j)=\frac{1}{2 \pi} \int_{0}^{\pi} \frac{\cos \varphi j}{\cos \varphi+\cosh z} d \varphi
$$

As $\cosh (z)>1$, the integrand does not have poles. According to Montroll and Potts (1955), Eq. (44) finally yields

$$
g(j)=\frac{(-1)^{j}}{2 \sinh z} e^{-|j| z}
$$

As the dislocation core is localized, it is reasonable to assume that the index $l$ in Eq. (39) only includes atoms in the core rather than in the entire system. Thus, Eq. (39) can be expressed in a matrix form as

$$
\mathbf{v}=\mathbf{T} \mathbf{v} .
$$

If we define an eigenvector and eigenvalue of $\mathbf{T}$ in mode $\mu$ as $\Psi^{\mu}$ and $\beta^{\mu}$, respectively, then

$$
\mathbf{T} \Psi^{\mu}=\beta^{\mu} \Psi^{\mu}
$$

is satisfied. Moreover, if we express $\mathbf{v}$ in terms of the basis $\boldsymbol{\Psi}^{\mu}$ as $\mathbf{v}=\sum_{\mu} v^{\mu} \boldsymbol{\Psi}^{\mu}$, then Eq. (46) is modified to $\sum_{\mu} v^{\mu}\left(1-\beta^{\mu}\right) \boldsymbol{\Psi}^{\mu}=0$. For Eq. (46) to have solutions, $\beta^{\mu}=1$ must be satisfied for all eigenmodes $\mu$.

As shown in Fig. 5, we assume that the center of the core is at the origin and $2 \sigma_{G}+1$ atoms are geometrically defined as the dislocation core. The core atoms are connected by a spring whose constant is $\gamma$ ' but the others are connected by $\gamma$. If we define an atomic

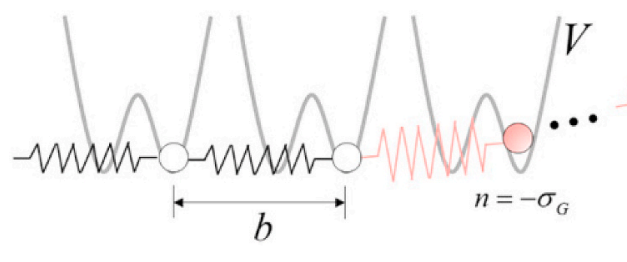

Phase 2

(Slipped)

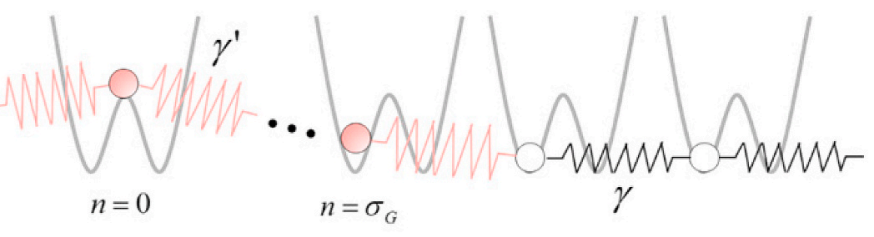

Phase 1

(Non-slipped)

Fig. 5. Discrete lattice model for dislocation oscillation. All the atoms are attached to substrate potential, $V$. As the dislocation glides to the right, the atoms moves to phase 2 , or neighboring valley, from phase 1 . The shaded atoms represent the dislocation core whose radius is $\sigma_{G} b$, and they are connected by an elastic spring whose stiffness is $\gamma$ '. But atoms outside of the core region are connected by the spring whose stiffness is $\gamma$. 
mass as $m$, the equations of motion are given by

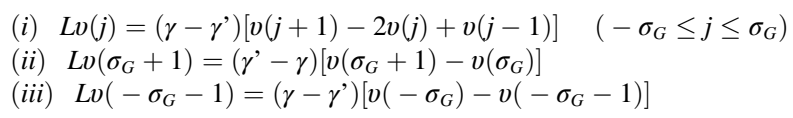

By comparing Eq. (48) with Eq. (33), the following relations can be obtained:

$$
\begin{aligned}
& \text { (i) } w^{(-1)}(j-1)=w^{(1)}(j+1)=\gamma-\gamma^{\prime}, w^{(0)}(j)=-2\left(\gamma-\gamma^{\prime}\right) \quad\left(-\sigma_{G} \leq j \leq-\sigma_{G}\right) \\
& \text { (ii) } w^{(-1)}\left(\sigma_{G}\right)=\gamma-\gamma^{\prime}, w^{(0)}\left(\sigma_{G}+1\right)=\gamma^{\prime}-\gamma \\
& \text { (iii) } w^{(-1)}\left(-\sigma_{G}-1\right)=\gamma^{\prime}-\gamma, w^{(0)}\left(-\sigma_{G}\right)=\gamma-\gamma
\end{aligned}
$$

By substituting the relations Eq. (49) into Eq. (39), Eq. (50) is derived.

$$
\begin{gathered}
v(j)=\gamma^{-1}\left[\left(\gamma-\gamma^{\prime}\right) g\left(j+\sigma_{G}\right)+\left(\gamma-\gamma^{\prime}\right) g\left(j+\sigma_{G}+1\right)\right] v\left(-\sigma_{G}-1\right) \\
+\gamma^{-1}\left[\left(\gamma-\gamma^{\prime}\right) g\left(j+\sigma_{G}-1\right)-2\left(\gamma-\gamma^{\prime}\right) g\left(j+\sigma_{G}\right)+\left(\gamma-\gamma^{\prime}\right) g\left(j+\sigma_{G}+1\right)\right] v\left(-\sigma_{G}\right) \\
+\cdots \gamma^{-1}\left[\left(\gamma-\gamma^{\prime}\right) g\left(j-\sigma_{G}-1\right)-2\left(\gamma-\gamma^{\prime}\right) g\left(j-\sigma_{G}\right)+\left(\gamma-\gamma^{\prime}\right) g\left(j-\sigma_{G}+1\right)\right] v\left(\sigma_{G}\right) \\
+\gamma^{-1}\left[\left(\gamma^{\prime}-\gamma\right) g\left(j-\sigma_{G}-1\right)+\left(\gamma-\gamma^{\prime}\right) g\left(j-\sigma_{G}\right)\right] v\left(\sigma_{G}+1\right) .
\end{gathered}
$$

If we define $P \equiv \gamma^{\prime} / \gamma$, then Eq. (50) is simplified to

$$
\begin{gathered}
v(j)=(1-P)\left[g\left(j+\sigma_{G}\right)-g\left(j+\sigma_{G}+1\right)\right] v\left(-\sigma_{G}-1\right) \\
+(1-P)\left[g\left(j-\sigma_{G}\right)-g\left(j-\sigma_{G}-1\right)\right] v\left(\sigma_{G}+1\right) \\
+\sum_{n=-\sigma_{G}}^{\sigma_{G}}[(1-P)\{g(j-n-1)+g(j-n+1)\}-2(1-P) g(j-n)] v(n)
\end{gathered}
$$

Here, the $\Sigma$ term that represents the displacement inside the core should be carefully considered because of the characteristic of the Green's function. By substituting the Green's function into Eq. (32),

$$
\begin{aligned}
& L g(j)=m \omega^{2} g(j)+\gamma[g(j+1)-2 g(j)+g(j-1)] \\
& \quad=\gamma\left[4 f^{2} g(j)+\{g(j+1)-2 g(j)+g(j-1)\}\right] .
\end{aligned}
$$

is derived. Moreover, by using Eq. (34),

$$
g(j+1)-2 g(j)+g(j-1)=-4 f^{2} g(j)
$$

is satisfied for $j \neq 0$ and

$$
4 f^{2} g(0)+g(1)-2 g(0)+g(-1)=1
$$

is satisfied for $j=0$. Then, the $\Sigma$ term in Eq. (51) follows

$$
(1-P)\{g(j-n-1)+g(j-n+1)\}-2(1-P) g(j-n)=4 f^{2}(P-1) g(j-n)
$$

if $j \neq n$ and

$$
(1-P)\{g(j-n-1)+g(j-n+1)\}-2(1-P) g(j-n)=(1-P)\left(-4 f^{2} g(0)+1\right)
$$

when $j=n$. Thus, Eq. (51) becomes

$$
\begin{gathered}
v(j)=(1-P)\left[g\left(j+\sigma_{G}\right)-g\left(j+\sigma_{G}+1\right)\right] v\left(-\sigma_{G}-1\right) \\
+(1-P)\left[g\left(j-\sigma_{G}\right)-g\left(j-\sigma_{G}-1\right)\right] v\left(\sigma_{G}+1\right)-4 f^{2}(1-P) \sum_{m=-\sigma_{G}}^{\sigma_{G}} g(j-m) v(m)
\end{gathered}
$$

if $j<-\sigma_{G}$ or $j>\sigma_{G}$ and it represents the phonon coordinate outside the core. Then, Eq. (51) becomes

$$
\begin{aligned}
& v(j)=(1-P)\left[g\left(j+\sigma_{G}\right)-g\left(j+\sigma_{G}+1\right)\right] v\left(-\sigma_{G}-1\right)+(1-P)\left[g\left(j-\sigma_{G}\right)-g\left(j-\sigma_{G}-1\right)\right] \\
& v\left(\sigma_{G}+1\right)-4 f^{2}(1-P) \sum_{\substack{m=-\sigma_{G} \\
(m \neq j)}}^{\sigma_{G}} g(j-m) v(m)+(1-P)\left[-4 f^{2} g(0)+1\right] v(j)
\end{aligned}
$$

when $-\sigma_{G} \leq j \leq \sigma_{G}$, and thus, it represents the phonon coordinate inside the core. Now, Eq. (56-1) and Eq. (56-2) can be expressed by the matrix equation in Eq. (57) by using the relation $g(-j)=g(j)$. 


$$
\begin{gathered}
{\left[\begin{array}{c}
v\left(-\sigma_{G}-1\right) \\
v\left(-\sigma_{G}\right) \\
\vdots \\
v\left(\sigma_{G}\right) \\
v\left(\sigma_{G}+1\right)
\end{array}\right]=\left[\begin{array}{ccc}
(1-P)[g(1)-g(0)] & 4 f^{2}(P-1) g(1) & \ldots \\
(1-P)[g(0)-g(1)] & 4 f^{2}(P-1) g(0)+(1-P) & \\
\vdots & \vdots & \ddots \\
(1-P)\left[g\left(2 \sigma_{G}\right)-g\left(2 \sigma_{G}+1\right)\right] & 4 f^{2}(P-1) g\left(2 \sigma_{G}\right) & \\
(1-P)\left[g\left(2 \sigma_{G}+1\right)-g\left(2 \sigma_{G}+2\right)\right] & 4 f^{2}(P-1) g\left(2 \sigma_{G}+1\right) & \ldots \\
4 f^{2}(P-1) g\left(2 \sigma_{G}+1\right) & (1-P)\left[g\left(2 \sigma_{G}+1\right)-g\left(2 \sigma_{G}+2\right)\right] \\
4 f^{2}(P-1) g\left(2 \sigma_{G}\right) & (1-P)\left[g\left(2 \sigma_{G}\right)-g\left(2 \sigma_{G}+1\right)\right] \\
\vdots & \vdots \\
4 f^{2}(P-1) g(0)+(1-P) & (1-P)[g(0)-g(1)] \\
4 f^{2}(P-1) g(1) & (1-P)[g(1)-g(0)]
\end{array}\right]\left[\begin{array}{c}
v\left(-\sigma_{G}-1\right) \\
v\left(-\sigma_{G}\right) \\
\vdots \\
v\left(\sigma_{G}\right) \\
v\left(\sigma_{G}+1\right)
\end{array}\right]}
\end{gathered}
$$

Here, the size of $\mathbf{T}$ is $\left(2 \sigma_{G}+3\right) \times\left(2 \sigma_{G}+3\right)$; hence, it has $2 \sigma_{G}+3$ eigenmodes. Thus, the number of oscillation eigenmodes depends on the size of the dislocation core. In other words, as the size of the dislocation core increases, the system can have more oscillation modes, although the size of the system does not change.

The eigenvectors of $\mathbf{T}$ are numerically obtained in the following section. However, without solving the exact eigenvalue problems, Eq. (56-1) and Eq. (56-2) can be further simplified due to the symmetric and anti-symmetric properties of the eigenmodes. Here, $2 \sigma_{G}+$ 3 eigenmodes are classified into two types, except for a single rigid mode. Consequently, $\sigma_{G}+1$ modes correspond to the symmetric mode and the remaining $\sigma_{G}+1$ modes correspond to the anti-symmetric mode. And since the single rigid mode has a zero eigenvalue and it does not contribute to the oscillation, it is ignored in this study. The eigenvectors of the anti-symmetric and symmetric modes can be described by

$$
\begin{aligned}
& \boldsymbol{\Psi}^{\mu, a}=\left[\begin{array}{llllll}
-a_{\sigma_{G}+1} & -a_{\sigma_{G}} \cdots & 0 & \cdots & a_{\sigma_{G}} & a_{\sigma_{G}+1}
\end{array}\right]^{T}, \\
& \boldsymbol{\Psi}^{\mu, s}=\left[\begin{array}{llllll}
s_{\sigma_{G}+1} & s_{\sigma_{G}} & \cdots & s_{0} & \cdots & s_{\sigma_{G}} \\
s_{\sigma_{G}+1}
\end{array}\right]^{T},
\end{aligned}
$$

respectively. Moreover, both Eq. (58) and Eq. (59) are normalized, or $\left|\Psi^{\mu, a}\right|=\left|\Psi^{\mu, s}\right|=1$. Physically, the eigenvector in mode $\mu$ indicates an array of the normalized phonon coordinates of the core atoms in $\left[-\sigma_{G}-1, \sigma_{G}+1\right]$ in the corresponding mode because Eq. (46) has a solution only when the eigenvalue of $\mathbf{T}$ is equal to 1 . Thus, $\mathbf{v}^{\mu}=\mathbf{T} \boldsymbol{\Psi}^{\mu}=\boldsymbol{\Psi}^{\mu}$ for the core atoms in $\left[-\sigma_{G}-1, \sigma_{G}+1\right]$. Moreover, according to Eq. (56-1), a phonon coordinate of an atom outside the dislocation core can be described as a linear combination of the phonon coordinates of the core atoms. Consequently, the phonon coordinates in mode $\mu$ for $j>\sigma_{G}+1$ can be derived by substituting Eq. (58) and Eq. (59) into Eq. (56-1), depending on the characteristic of each eigenmode. Thus,

$$
\begin{gathered}
\frac{v^{\mu, a}(j)}{v_{0}^{\mu, a}}=(1-P) \frac{(-1)^{j+\sigma_{G}}}{2 \sinh z}\left(1+e^{-z}\right)\left(-e^{-\left(j+\sigma_{G}\right) z}+e^{-\left(j-\sigma_{G}-1\right) z}\right) a_{\sigma_{G}+1} \\
-4 f^{2}(1-P)\left[\sum_{n=1}^{\sigma_{G}} \frac{(-1)^{j-n}}{2 \sinh z} e^{-(j-n) z} a_{n}+\sum_{n=-\sigma_{G}}^{-1} \frac{(-1)^{j-n}}{2 \sinh z} e^{-(j-n) z} a_{n}\right],
\end{gathered}
$$

where $v_{0}^{\mu, a}$ is the reference amplitude of the anti-symmetric oscillation mode $\mu$, is derived for the anti-symmetric mode, and

$$
\begin{gathered}
\frac{v^{\mu, s}(j)}{v_{0}^{\mu, s}}=(1-P) \frac{(-1)^{j+\sigma_{G}}}{2 \sinh z}\left(1+e^{-z}\right)\left(e^{-\left(j+\sigma_{G}\right) z}+e^{-\left(j-\sigma_{G}-1\right) z}\right) s_{\sigma_{G}+1} \\
-4 f^{2}(1-P) \frac{(-1)^{j}}{2 \sinh z}\left[\sum_{n=1}^{\sigma_{G}}(-1)^{n}\left(e^{-(j-n) z}+e^{-(j+n) z}\right) s_{n}+e^{-j z} s_{0}\right],
\end{gathered}
$$

where $v_{0}^{\mu, s}$ is the reference amplitude of the symmetric oscillation mode $\mu$, and is derived for the symmetric mode. Both Eq. (60) and Eq. (61) are further simplified to

$$
\begin{aligned}
& \frac{v^{\mu, a}(j)}{v_{0}^{\mu, a}}=\frac{2 f(P-1)}{\sinh z}(-1)^{j} e^{-j z}\left[2 f \sum_{n=1}^{\sigma_{G}}(-1)^{n} \sinh (n z) a_{n}-(-1)^{\sigma_{G}} \sinh \left(\sigma_{G}+\frac{1}{2}\right) z a_{\sigma_{G}+1}\right], \\
& \frac{v^{\mu, s}(j)}{v_{0}^{\mu, s}}=\frac{2 f(P-1)}{\sinh z}(-1)^{j} e^{-j z}\left[2 f \sum_{n=1}^{\sigma_{G}}(-1)^{n} \cosh (n z) s_{n}+f s_{0}-(-1)^{\sigma_{G}} \cosh \left(\sigma_{G}+\frac{1}{2}\right) z s_{\sigma_{G}+1}\right],
\end{aligned}
$$

respectively. Furthermore, through the same procedure used to derive Eq. (62) and Eq. (63), we obtain $v^{\mu, a}(j)=-v^{\mu, a}(-j)$ and $v^{\mu, s}(j)=$ $v^{\mu, s}(-j)$ for $j<-\sigma_{G}-1$.

\subsection{Drag force}

As we derived phonon coordinates for both the anti-symmetric and symmetric modes in the preceding section, we now derive the phonon drag force. Both Eq. (62) and Eq. (63) can be described as functions of the distance $r=j b$ as

$$
v^{\mu, a}(r)=V_{0}^{\mu, a}\left(\sigma_{G}, z\right) e^{-\frac{z}{b} r}, \quad v^{\mu, a}(-r)=-v^{\mu, a}(r),
$$




$$
v^{\mu, s}(r)=V_{0}^{\mu, s}\left(\sigma_{G}, z\right) e^{-\frac{z}{b} r}, \quad v^{\mu, s}(-r)=v^{\mu, s}(r)
$$

for the anti-symmetric and symmetric modes, respectively. Furthermore, their coefficients depend on the geometric parameters of the dislocation core. By substituting Eq. (64) and Eq. (65) into Eq. (29), the drag forces in mode $\mu$ are derived as Eq. (66) and Eq. (67) for the anti-symmetric and symmetric modes, respectively.

$$
\begin{gathered}
F_{\text {drag }}^{\mu, a}=-\frac{b \tau_{a p p}}{\sigma_{D}^{3} \sqrt{2 \pi}} \int_{-R}^{R} r v(r) e^{-\frac{r^{2}}{2 \sigma_{D}^{2}}} d r, \\
F_{\text {drag }}^{\mu, s}=-\frac{G b^{2}}{\sigma_{D}^{3} 4 \pi \sqrt{2 \pi}} \int_{-R}^{R} v(r) e^{-\frac{r^{2}}{2 \sigma_{D}^{2}}} d r .
\end{gathered}
$$

Through integration by parts, the integral term in Eq. (66) becomes

$$
\int_{-R}^{R} r v(r) e^{-\frac{r^{2}}{2 \sigma_{D}^{2}}} d r=2 V_{0}^{\mu, a} \int_{0}^{R} r e^{-\frac{r^{2}}{2 \sigma_{D}^{2}}} \cdot e^{-\frac{z r}{b}} d r=2 V_{0}^{\mu, a}\left[\sigma_{D}^{2}\left(1-e^{-\left(\frac{R^{2}}{2 \sigma_{D}^{2}}+\frac{z R}{b}\right)}\right)-\sqrt{\frac{\pi}{2}} \frac{\sigma_{D}^{3} z}{b} e^{\frac{\sigma_{D}^{2} z^{2}}{2 b^{2}}}\left[\operatorname{erf}\left(t_{R}\right)-\operatorname{erf}\left(t_{0}\right)\right]\right]
$$

where

$$
\begin{aligned}
& t_{0}=\frac{\sigma_{D} z}{\sqrt{2} b}, \\
& t_{R}=\frac{1}{\sqrt{2} \sigma_{D}}\left(R+\frac{\sigma_{D}^{2} z}{b}\right)
\end{aligned}
$$

Thus, by substituting Eq. (68) into Eq. (66), the drag force for the anti-symmetric mode is finally derived as

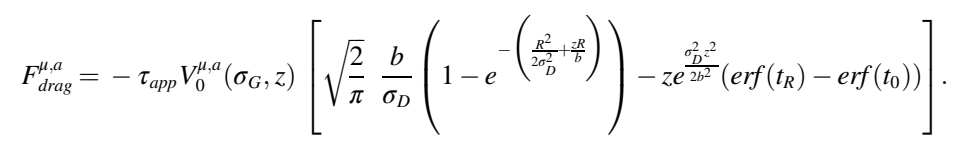

And the integral term in Eq. (67) becomes

$$
\int_{-R}^{R} v(r) e^{-\frac{r^{2}}{2 \sigma_{D}^{2}}} d r=\sqrt{2 \pi} V_{0}^{\mu, s} \sigma_{D} e^{\frac{\sigma_{D}^{2} z^{2}}{2 b^{2}}}\left(\operatorname{erf}\left(t_{R}\right)-\operatorname{erf}\left(t_{0}\right)\right) .
$$

By substituting Eq. (71) into Eq. (67), the drag force for the symmetric mode is finally derived as

$$
F_{\text {drag }}^{\mu, s}=-\frac{G V_{0}^{\mu, s}\left(\sigma_{G}, z\right)}{4 \pi}\left(\frac{b}{\sigma_{D}}\right)^{2} e^{\frac{\sigma_{D}^{2} z^{2}}{2 b^{2}}}\left(\operatorname{erf}\left(t_{R}\right)-\operatorname{erf}\left(t_{0}\right)\right) .
$$

From Eq. (70) and Eq. (72), we can conclude that the anti-symmetric phonon modes only interact with the external strain field, whereas the symmetric modes only interact with the self-strain field caused by a dislocation. Furthermore, both equations show that the drag force is a function of the integration radius; consequently, the $J$ integral is path-dependent, unless the radius is sufficiently large.

According to Eq. (70) and Eq. (72), the drag force for both eigenmodes depends on the geometric and mechanical properties of the dislocation core. For example, as $P \rightarrow 1$, which indicates that the core atoms are replaced by perfect atoms, both $V_{0}^{\mu, a}\left(\sigma_{G}, z\right)$ and $V_{0}^{\mu, s}\left(\sigma_{G}, z\right)$ become zero so that the drag force disappears. Furthermore, the drag force of mode $\mu$, which is integrated along a sufficiently large path, will increase with increasing $P$ because $V_{0}^{\mu, a}$ (or $V_{0}^{\mu, s}$ ) increases. This indicates that more energy dissipates from the dislocation core as it becomes stiffer, because a stiffer core increases the oscillation frequency. Consequently, the drag force makes the $J$ integral depend on the properties of the dislocation core. This indicates that a parametric study is required to investigate the influence of the properties of the core on the $J$ integral during the dislocation glide.

\section{Results}

In the preceding section, we analytically derived the drag force for the anti-symmetric and symmetric eigenmodes. In this section, we numerically solve Eq. (57) to obtain eigenvectors and use them to calculate the drag force through Eq. (30). 


\subsection{Eigenmode analysis}

Among $2 \sigma_{G}+2$ eigenvalues of $\mathbf{T}$, except $\beta=0$, which corresponds to the rigid mode, $\sigma_{G}+1$ eigenvalues represent the antisymmetric modes and $\sigma_{G}+1$ eigenvalues represent the symmetric modes. However, for Eq. (57) to have solutions, it is necessary to determine the values of $f^{\mu}$ and $P^{\mu}$ that make $\beta^{\mu}=1$. This constraint makes $f^{\mu}$ and $P^{\mu}$ uniquely related. After determining the relationship between $f^{\mu}$ and $P^{\mu}$, the corresponding eigenvector $\Psi^{\mu}$ is obtained. Especially, if $\sigma_{G}=0$ so that the dislocation is characterized by a point defect, the relation between $f^{\mu}$ and $P^{\mu}$ for $\sigma_{G}=0$ can be analytically obtained. According to Montroll and Potts (1955), the symmetric mode is given by

$$
f=\sqrt{\frac{P(3 P-4)+P \sqrt{P(9 P-8)}}{8(P-1)}},
$$

and the anti-symmetric mode is given by

$$
f=\frac{P}{\sqrt{4(P-1)}}
$$

The comparison between the analytical solutions and our numerical solutions for $\sigma_{G}=0$ is graphically shown in Fig. 6. It can be observed that our numerical algorithm exactly reproduced the analytical solutions for both eigenmodes.

Now, we apply the numerical method to solve Eq. (57) for cases with $\sigma_{G}>0$, or when the dislocation core has a finite width, and determine the relations between $f^{\mu}$ and $P^{\mu}$ by solving Eq. (47) with $\beta^{\mu}=1$. The relations between $f^{\mu}$ and $P^{\mu}$ for $\sigma_{G}=1,2$, and 3 are graphically illustrated in Fig. 7(a), (b), and 7(c), respectively. Furthermore, their normalized eigenvectors depending on the oscillation frequency are shown in Figs. 8-10, respectively. According to Fig. 7(a), (b), and 7(c), the number of activated eigenmodes depends on $P^{\mu}$. As an example, for $\sigma_{G}=1$, only a single symmetric mode is activated when $1.0<P^{\mu}<1.3$, and one symmetric and one antisymmetric modes are activated when $1.3 \leq P^{\mu}<2.2$. Two symmetric and one anti-symmetric modes are activated when $2.2 \leq P^{\mu}<$ 7.3. Finally, all the possible eigenmodes are activated only when $P^{\mu} \geq 7.3$. This is equally applied for $\sigma_{G}=2,3$, and even larger values. This difference in eigenmode activation is due to the difference in $P^{\mu}$ that satisfies $\beta^{\mu}=1$ for each mode. In Fig. 7(a), (b), and 7(c), the $P^{\mu}$ required to activate the next eigenmode drastically increases as the number of currently activated modes increases, and the newly generated mode occupies the lowest frequency or the lowest energy level. Notably, the symmetric and anti-symmetric modes are alternatively activated. However, as the symmetric mode is always generated first, the number of symmetric modes is always greater than or equal to that of anti-symmetric modes.

Additional information can be obtained from the eigenvectors of each mode shown in Figs. 8-10. For $\sigma_{G}=1$, comparing the symmetric eigenvectors in Fig. 8(a)-(c), the latter has a smaller frequency but a larger absolute value at the edge elements than the former. A similar tendency is observed when the anti-symmetric eigenvectors in Fig. 8(b)-(d) are compared. For $\sigma_{G}=2$, comparing the symmetric eigenvectors in Fig. 9(a), (c), and 9(e), the distribution of Fig. 9(a) is the most centralized but that of Fig. 9(e) is largely localized at the edge atoms, or at the atoms whose numbers are \pm 3 . Furthermore, as the anti-symmetric eigenvector changes from Fig. 9(b)-9(f), its distribution becomes more localized at the edge atoms, as in the symmetric mode. The same tendency is observed for

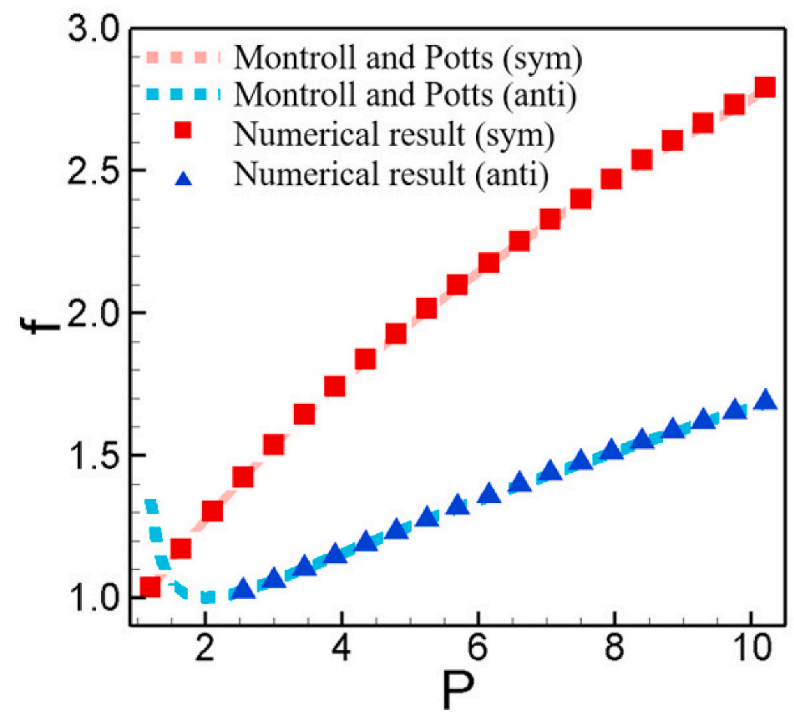

Fig. 6. Relation between $P\left(=\gamma^{\prime} / \gamma\right)$ and $f\left(=\omega / \omega_{L}\right)$ for $\sigma_{G}=0$, or a point defect case. The red and blue dashed lines represent Eqs. (73) and (74) derived by Montroll and Potts (1955), respectively. The red squares and blue triangles represent the numerical results obtained by solving Eq. (47) with $\beta^{\mu}=1$. They correspond to the symmetric and anti-symmetric modes, respectively. 
(a)

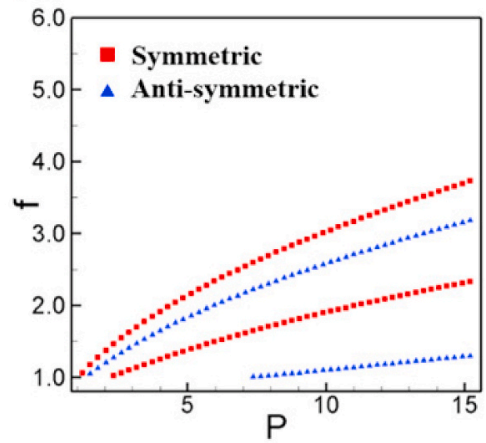

(b)

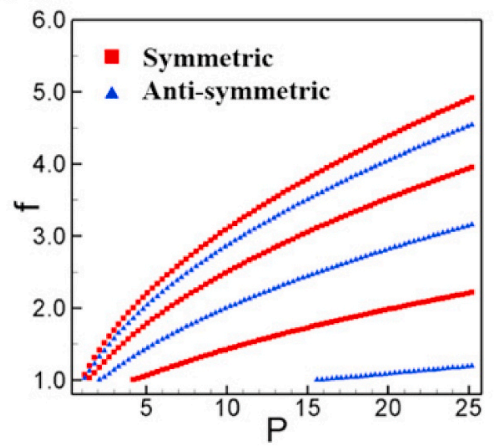

(c)

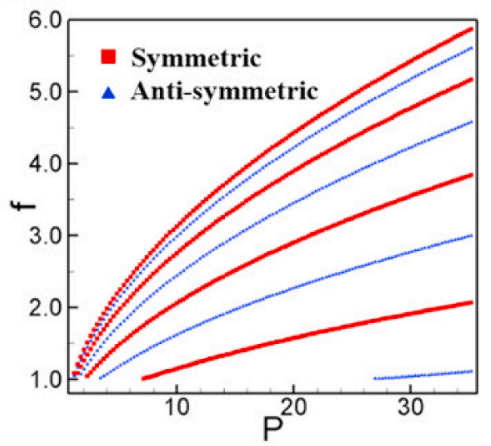

Fig. 7. Relation between $P$ and $f$ for (a) $\sigma_{G}=1$, (b) $\sigma_{G}=2$, and (c) $\sigma_{G}=3$, which are obtained by solving Eq. (47) with $\beta^{\mu}=1$. The red squares represent the symmetric modes and the blue triangles represent the anti-symmetric modes.

(a)

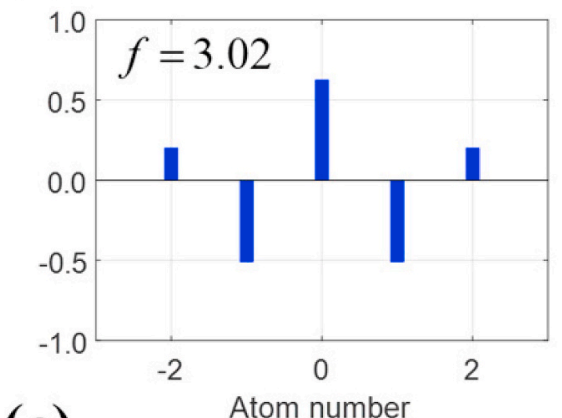

(c)

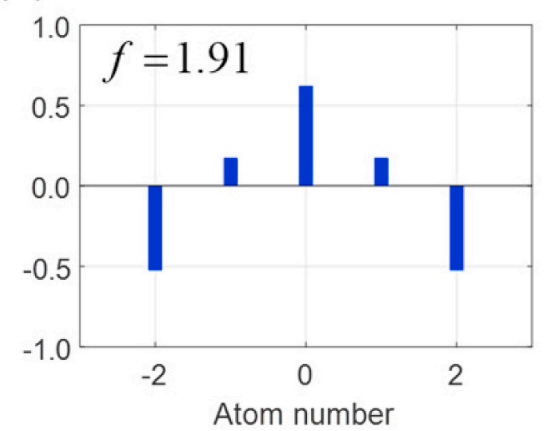

(b)
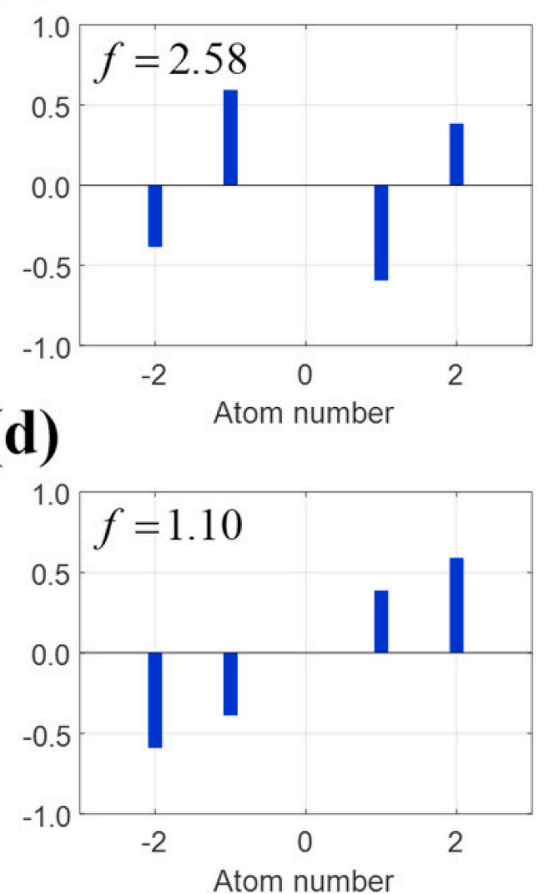

Fig. 8. Normalized eigenvectors for $\sigma_{G}=1$ with $P=10$. (a) and (c) are symmetric modes and (b) and (d) are anti-symmetric modes.

both the symmetric and anti-symmetric modes in Fig. 10. This proves that the frequency of the eigenmode increases as the element distribution of its eigenvector within the core is more concentrated at its center. Therefore, the eigenmode analysis shows that there is a consistent relationship between the frequency and displacement distribution of the atoms in the dislocation core. This is because all the eigenmodes share the same eigenvalue, or $\beta^{\mu}=1$.

\subsection{Drag force analysis}

The contribution of each eigenmode to the drag force was investigated by changing the properties of the dislocation core. First, the phonon coordinates were separately calculated for the core atoms and the atoms outside the core. The phonon coordinates of the core atoms, or atom $j$ for $j \in\left[-\sigma_{G}-1, \sigma_{G}+1\right]$, correspond to the elements of the eigenvector in mode $\mu$. The phonon coordinates of the atoms outside the core in the same mode were obtained by using Eq. (62) and Eq. (63). Subsequently, the drag force was numerically calculated by substituting the obtained phonon coordinates into Eq. (30). $G=71.5 \mathrm{GPa}$ and $b=2.473 \AA$ are used as the input 
(a)

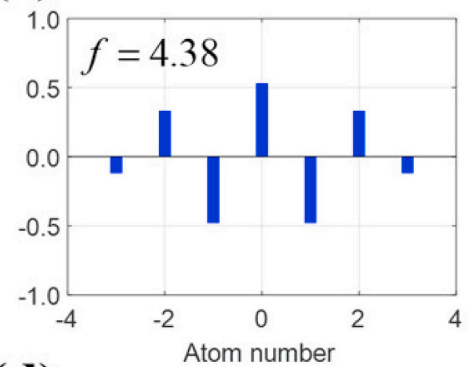

(d)

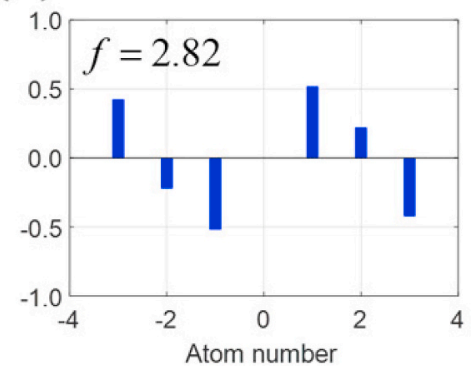

(b)

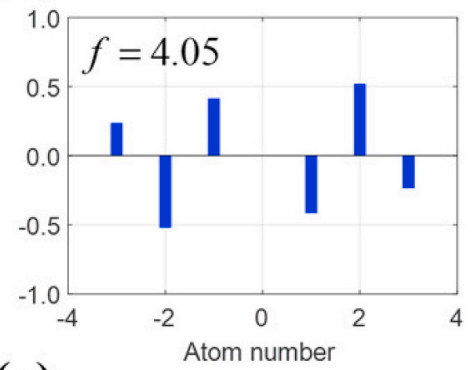

(e)

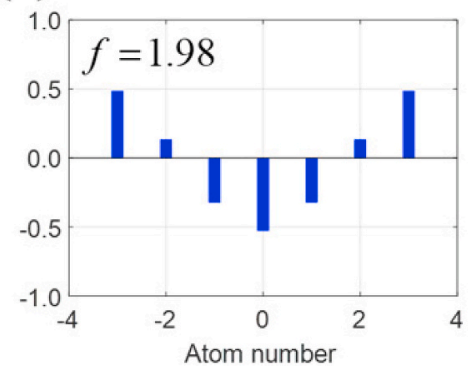

(c)

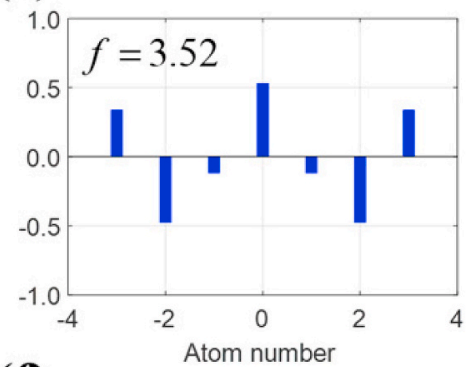

(f)

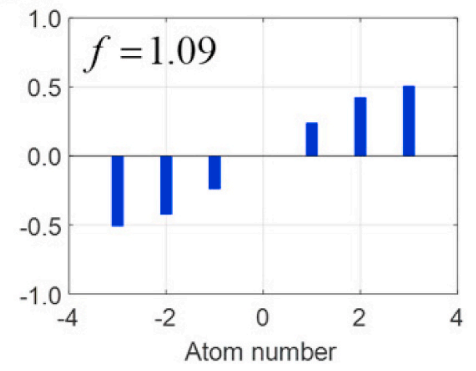

Fig. 9. Normalized eigenvectors for $\sigma_{G}=2$ with $P=20$. (a), (c), and (e) are symmetric modes and (b), (d), and (f) are anti-symmetric modes.

(a)

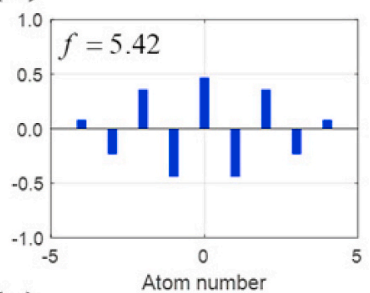

(e)

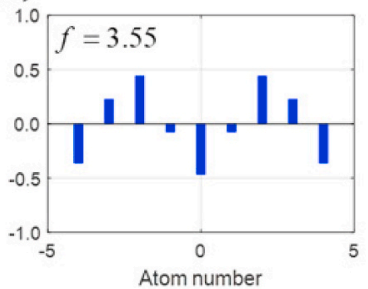

(b)

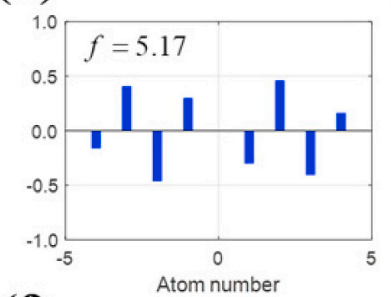

(f)

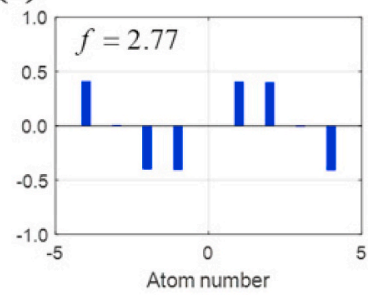

(c)

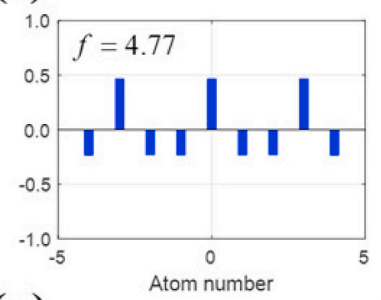

(g)

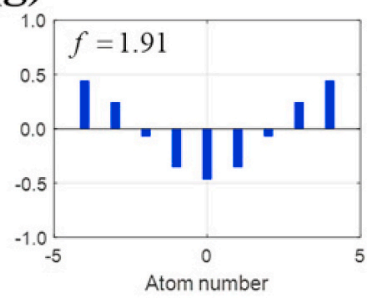

(d)

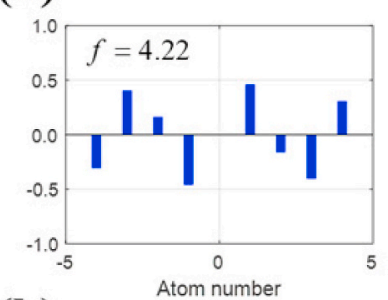

(h)

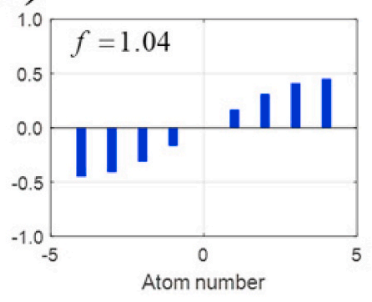

Fig. 10. Normalized eigenvectors for $\sigma_{G}=3$ with $P=30$. (a), (c), (e), and (g) are symmetric modes and (b), (d), (f), and (h) are antisymmetric modes.

parameters to calculate the drag force (Kim et al., 2020). Additionally, we assumed $\sigma_{D}=\sigma_{G} b$ and $v_{0}^{\mu, a}=v_{0}^{\mu, s}=b$ for simplicity. In this study, the resulting drag force was normalized by $\tau_{a p p} b$, which is the PK force. The normalized drag force as a function of $P$ for $\sigma_{G}=2$ (i. e., the general size of a dislocation core without dissociation in cubic crystals) is shown in Fig. 11. Here, the drag force was calculated by changing the integration radius $R$. Consequently, according to Fig. 11, the magnitude of the drag force increases with increasing $P$ in general and finally converges to a certain value for every mode. In particular, the subsequently activated (SA) symmetric and anti-symmetric modes cause a much larger drag force than the formerly activated (FA) symmetric and anti-symmetric modes at a given $P$, respectively. This can be intuitively explained by investigating the dependency of the analytically derived drag force on the eigenmode frequency. According to Eq. (70) and Eq. (72), if $R=N_{R} b$ is sufficiently large to surround all the core atoms with extra space, or $N_{R} \gg \sigma_{G}$, the drag forces for the anti-symmetric and symmetric modes, respectively, become approximately 
(a)

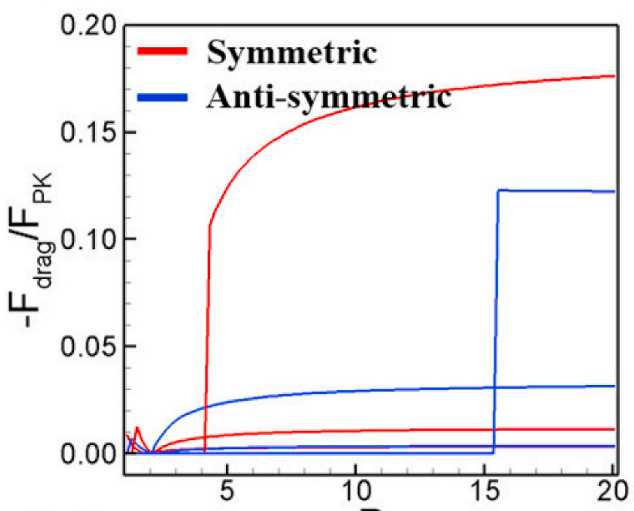

(c)

P

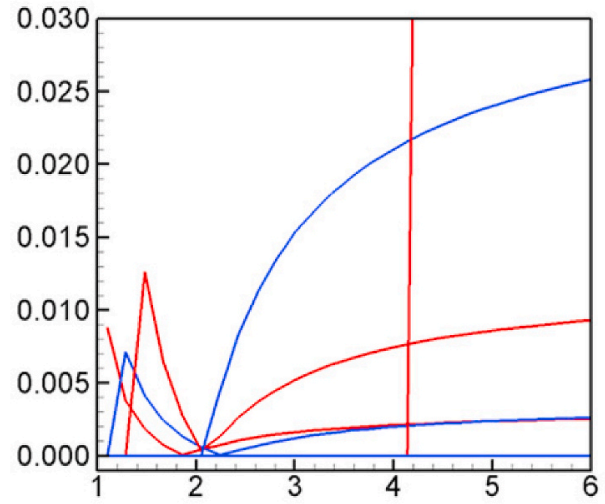

(b)
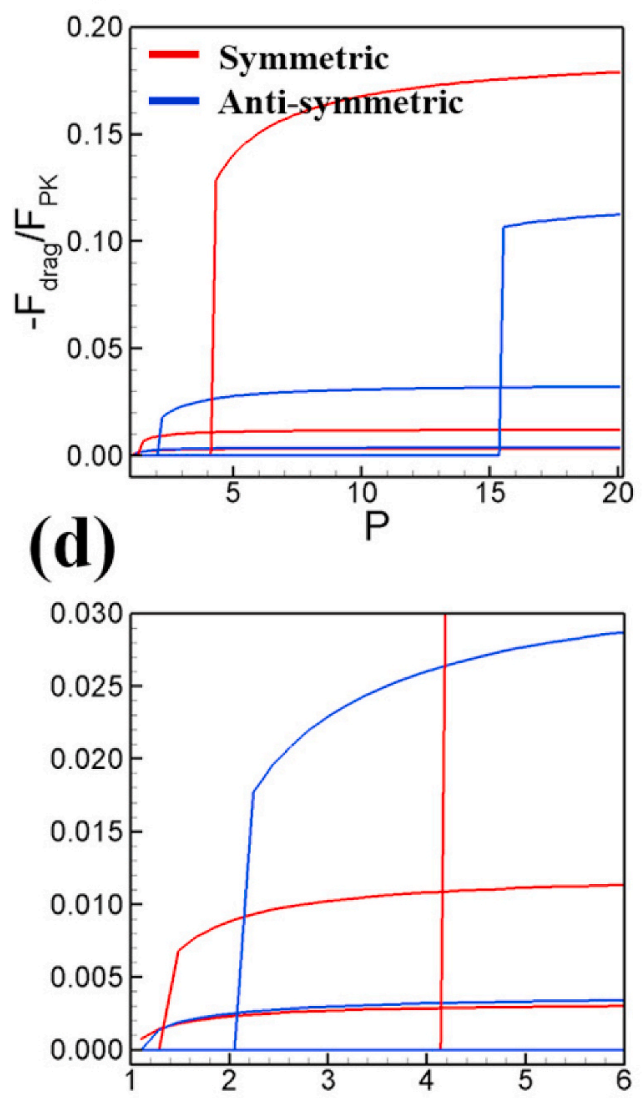

Fig. 11. Normalized drag force as a function of $P$ for $\sigma_{G}=2$ when (a) $R=3 b$ and (b) $R=9 b$. The red and blue lines represent the contributions of the symmetric and anti-symmetric eigenmodes, respectively. (c) and (d) are the enlarged figures of (a) and (b) for small $P$, respectively.

$$
\begin{aligned}
& \left|F_{\text {drag }}^{\mu, a}\right| \approx \tau_{a p p} V_{0}^{\mu, a}\left[\sqrt{\frac{2}{\pi}} \frac{b}{\sigma_{D}}-z e^{\frac{\sigma^{2} z^{2}}{2 b^{2}}}\left(1-\operatorname{erf}\left(t_{0}\right)\right)\right], \\
& \left|F_{\text {drag }}^{\mu, s}\right| \approx \frac{G}{4 \pi} V_{0}^{\mu, s}\left(\frac{b}{\sigma_{D}}\right)^{2} e^{\frac{\sigma_{D}^{2} z^{2}}{2 b^{2}}}\left(1-\operatorname{erf}\left(t_{0}\right)\right) .
\end{aligned}
$$

As the SA mode has a much lower frequency than the FA mode at a fixed $P$, it has a much smaller $z$. For the anti-symmetric modes, as Eq. (75) decreases with increasing $z$, the drag force is larger for the FA mode than for the SA mode. In particular, if $z$ is so large that $t_{0}>$ 2, the second term in Eq. (75) becomes much smaller than the first term. Therefore, the drag force becomes

$$
\left|F_{\text {drag }}^{\mu, a}\right| \approx \tau_{\text {app }} V_{0}^{\mu, a}\left(\sigma_{G}, z\right) \sqrt{\frac{2}{\pi}} \frac{b}{\sigma_{D}}
$$

and its dependence on the frequency is determined by $V_{0}^{\mu, a}\left(\sigma_{G}, z\right)$. By substituting Eq. (62) into Eq. (77), we obtain

$$
\left|\frac{F_{\text {drag }}^{\mu, a}}{\tau_{\text {app }} v_{0}^{\mu, a}}\right| \sim \frac{2 f}{\sinh z}\left[2 f \sum_{n} \sinh (n z) a_{n}\right] \sim \frac{2 f}{2 f \sqrt{f^{2}-1}}\left[2 f \sum_{n} \sinh (n z) a_{n}\right] \sim \sum_{n} \sinh (n z) a_{n}
$$

As the edge atoms of the dislocation core in the SA mode have larger eigenvector elements than those in the FA mode, the drag force is much larger for the SA mode than for the FA mode. For the symmetric mode, as $e^{\frac{\sigma_{D}^{2} z^{2}}{2 b^{2}}}\left[1-\operatorname{erf}\left(t_{0}\right)\right]$ in Eq. (76) is a decreasing function of $z$ and the same edge-localized effect occurs as in the anti-symmetric mode, the drag force is much larger for the SA mode than for the FA mode.

Interestingly, when $R$ is so small that it includes only the core atoms with a few additional atoms, a decrease in the drag force is 
observed in Fig. 11(a) and (c) with increasing $P$ at a small $P$. As $R$ is small, the drag force for both the anti-symmetric and symmetric modes is mainly determined by the eigenvectors of the core. From Fig. 12, it can be observed that the distribution of eigenvector elements becomes centralized as $P$ increases at a fixed eigenmode. This causes $V_{0}^{\mu, a}\left(\sigma_{G}, z\right)$ and $V_{0}^{\mu, s}\left(\sigma_{G}, z\right)$ to decrease with increasing $P$ and finally results in a decrease in the drag force. Consequently, the distribution of eigenvector elements within the dislocation core is crucial to determine the contribution of each mode to the drag force. However, at a large $P$, the variation of the eigenvector distribution is not large with changing $P$. Rather, $V_{0}^{\mu, a}\left(\sigma_{G}, z\right)$ and $V_{0}^{\mu, s}\left(\sigma_{G}, z\right)$ increase with increasing $P$ because they are proportional to $(P-1)$ according to Eq. (62) and Eq. (63). Consequently, the drag force is strongly influenced by the displacement distribution of the atoms of the dislocation core. Furthermore, according to Figs. 8-10, the displacement distribution inside the core strongly depends on the frequency of each eigenmode rather than the stiffness of the dislocation core. In general, the drag force increases with decreasing core frequency because the atomic displacements inside the core are gradually concentrated at the edge atoms as the core frequency becomes lower. This is reflected in terms of the hyperbolic function in Eq. (78).

In addition, we investigated the dependency of the drag force on the core width for both the anti-symmetric and symmetric modes. By using Eq. (75) and Eq. (76) with $z=1.0$, the relationship between the drag force and the core width can be computed. This is shown in Fig. 13. It can be observed that the magnitude of the drag force decreases as the core width increases. In other words, a larger drag force is induced during the motion of the dislocation as it has a more compact structure. This result is qualitatively identical to previous studies (Lee et al., 2011; Kim et al., 2020). According to a previous study (Kim et al., 2020), it was proved that the oscillation of a dislocation during its gliding motion dissipates energy around its core, which lowers the average stress around the moving dislocation. This is the source of the drag force, and it is quantified by Eq. (79) based on discrete lattice dynamics.

$$
\left|\frac{F_{\text {drag }}}{F_{P K}}\right|=C_{1}^{d i s}-\left(\frac{\omega_{L} b}{2 \pi v}\right)^{2} C_{2}^{d i s},
$$

where $v$ is the dislocation velocity. Here, $C_{1}^{\text {dis }}$ and $C_{2}^{\text {dis }}$ are defined, respectively, as

$$
\begin{aligned}
& C_{1}^{d i s}=\frac{2 B \Gamma_{d i s}}{\pi^{2} m \omega}, \\
& C_{2}^{d i s}=\frac{B \Gamma_{d i s} \omega}{m \omega_{L}^{2}},
\end{aligned}
$$

where $B$ is the drag coefficient. Furthermore, it was proved that $\Gamma_{\text {dis }}$ is a dimensionless constant proportional to the compactness of the dislocation core.

If the dislocation velocity is sufficiently high to satisfy $w_{L} b \ll 2 \pi v$, the dislocation oscillates with a high frequency, and this

(a)

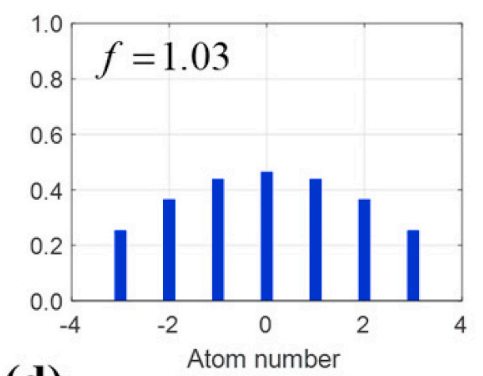

(d)

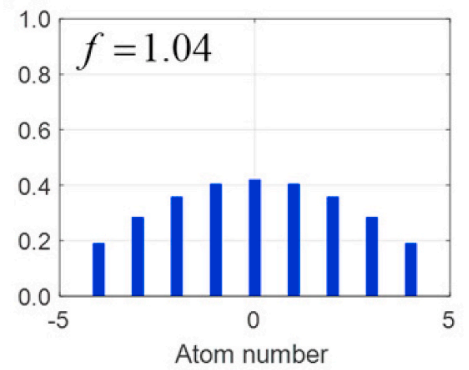

(b)

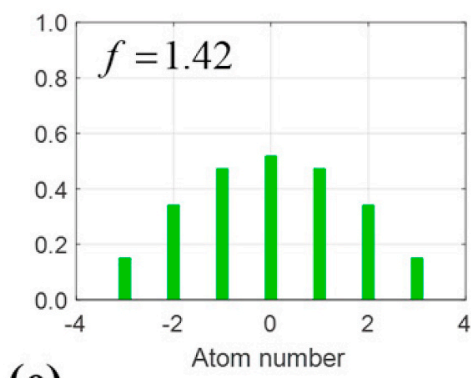

(e)

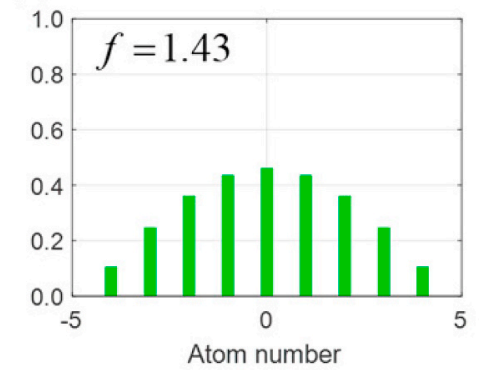

(c)

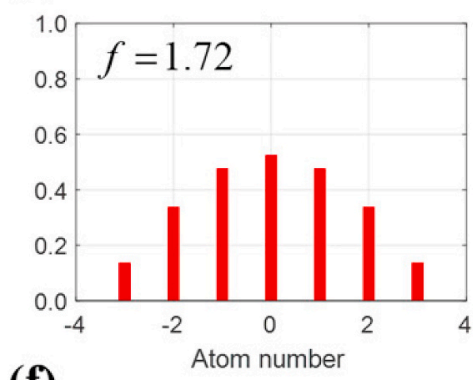

(f)

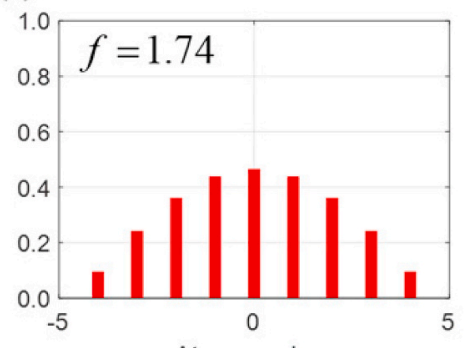

Atom number

Fig. 12. Absolute values of the normalized eigenvector in mode 1 with changing $P$. Each figure corresponds to the eigenvector (a) for $\sigma_{G}=2$ and $P=1.1$, (b) for $\sigma_{G}=2$ and $P=2.1$, (c) for $\sigma_{G}=2$ and $P=3.1$, (d) for $\sigma_{G}=3$ and $P=1.1$, (e) for $\sigma_{G}=3$ and $P=2.1$, and (f) for $\sigma_{G}=3$ and $P=$ 3.1. 


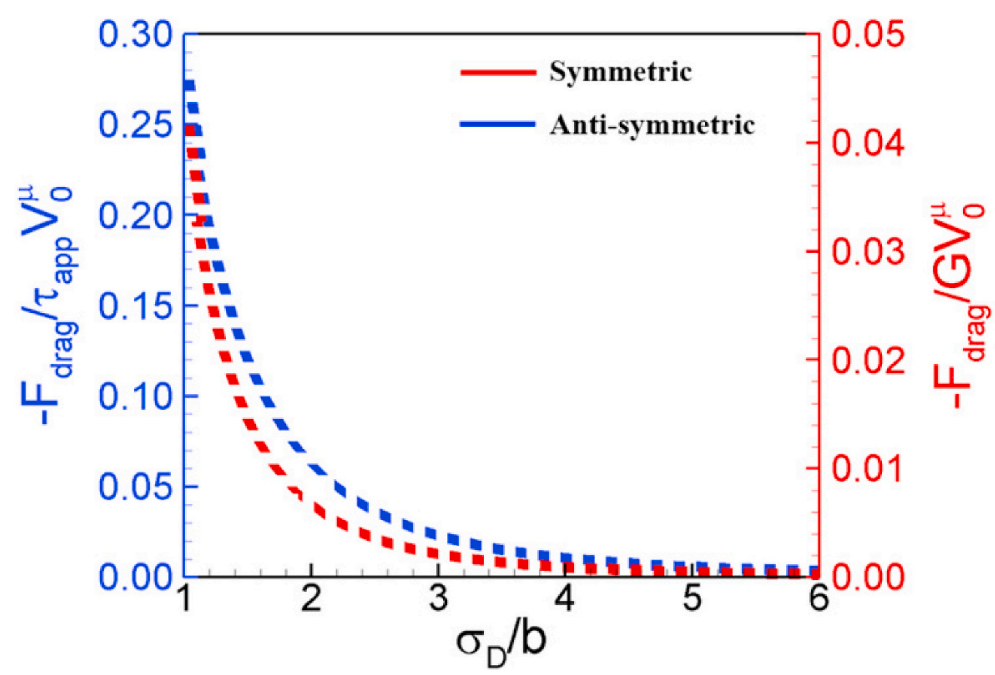

Fig. 13. Relationship between the normalized drag force and core width for symmetric and anti-symmetric modes. Here, $z=1.0$ is fixed for both modes.

oscillation dominates the relativistic drag (Kim et al., 2020). Consequently, Eq. (79) is simplified to $\left|F_{d r a g} / F_{P K}\right| \approx C_{1}^{\text {dis }}$. Therefore, increasing $\Gamma_{\text {dis }}$ leads to an increase in $F_{\text {drag }}$, which is the same as the result of this study. Furthermore, in our model, the anti-symmetric mode mainly contributes to the drag force in the limit of high frequency because erf $\left(t_{0}\right) \rightarrow 1$ according to Eq. (69-1). Thus, Eq. (81) is satisfied.

$$
\left|\frac{F_{\text {drag }}}{F_{P K}}\right|=\left|\frac{F_{\text {drag }}}{\tau_{\text {app }} b}\right| \approx \sqrt{\frac{2}{\pi}} \frac{V_{0}^{\mu, a}}{\sigma_{D}} .
$$

As $\left|F_{\text {drag }} / F_{P K}\right| \approx C_{1}^{\text {dis }}$, we can derive $C_{1}^{\text {dis }}$ as

$$
\begin{aligned}
& C_{1}^{d i s}=\sqrt{\frac{2}{\pi}} \frac{V_{0}^{\mu, a}\left(\sigma_{G}, z\right)}{\sigma_{D}} \\
& \sim \sqrt{\frac{2}{\pi}}\left(\frac{b}{\sigma_{D}}\right) \frac{2 f(P-1)}{\sinh z}\left[2 f \sum_{n=1}^{\sigma_{G}}(-1)^{n} \sinh (n z) a_{n}-(-1)^{\sigma_{G}} \sinh \left(\sigma_{G}+\frac{1}{2}\right) z a_{\sigma_{G}+1}\right] .
\end{aligned}
$$

Therefore, we conclude that the drag force is caused mainly by the anti-symmetric oscillation mode when the dislocation velocity is sufficiently high to be comparable to the sound wave velocity. Furthermore, the drag force is quantified by Eq. (82), in which the structural and mechanical properties of the dislocation core are well reflected.

In this work, we assumed $f>1$, which means that the dislocation vibration frequency is beyond the frequency band of the perfect lattice. In general, this type of oscillation occurs when the material is suffered by high strain rate or temperature (i.e. $\dot{\varepsilon} \geq \sim 10^{5} s^{-1}$ or $T \geq \theta_{D}$ ) because strong excitations are required for the dislocation to have such high frequency. This assumption leads the radiated phonons to be localized around the dislocation (Sun et al., 2019) and they form a viscous gas surrounding the dislocation. In addition, the relativistic effect is accompanied due to the extreme conditions and the resulting drag force depends on the amount of phonon localization. As a result, the dislocation motion disturbs the phonon gas to reach the equilibrium state and the dislocation is slowed down as it interacts with the phonon gas. Therefore, the phonon drag mechanism becomes the dominant drag mechanism for a fast-moving dislocation. The phonon drag is quite different from radiation damping that is dominant drag mechanism for very low temperature. While the extreme conditions are required for the phonon drag, the radiation damping can occur without it. Hence, the radiation damping becomes the dominant drag mechanism for dislocation whose oscillation frequency is within $0<f<1$. In this case, the phonons are scattered by the dislocation rather than localized around it.

\section{Discussion}

In previous sections, we have focused on physics of dislocation, and numerically calculated various properties of an oscillating dislocation whose frequency is beyond the frequency band of the perfect lattice. In this section, starting from dislocation velocity-stress relation for a single dislocation, we introduce applications of our theory to plasticity models. 


\subsection{Dislocation velocity-stress relation}

Considering that dislocation motion is a material response of external loads, the dislocation velocity-stress curve is one of the most important functions characterizing material plasticity. According to Kim et al. (2020), the velocity of fast-moving dislocation can be obtained by solving the below cubic equation:

$$
v^{3}-M_{0} \tau_{a p p} b\left(1-C_{1}^{d i s}\right)-\frac{M_{0} \tau_{a p p} \omega_{L}^{2} b^{3}}{4 \pi^{2}}\left(1-C_{2}^{d i s}\right)=0,
$$

where $M_{0}$ is instantaneous dislocation mobility at critical stress $\tau_{c}$ to move the dislocation and $C_{2}^{\text {dis }}=\left(\pi^{2} f^{2} / 2\right) C_{1}^{\text {dis }}$. By substituting Eq. (82) into Eq. (83) with given material properties, $v\left(\tau_{\text {app }}\right)$ can be obtained. Therefore, the solution of Eq. (83) represents a dislocation mobility law, which is differentiated from continuum-based one because discrete properties of the dislocation core can be characterized by $C_{1}^{\text {dis }}$ and $C_{2}^{\text {dis }}$. To obtain $v\left(\tau_{a p p}\right)$, we used $M_{0}, b$ and $\omega_{L}$ as input parameters of an edge dislocation in 2D triangular lattice, which were obtained by molecular dynamics (MD) simulation in previous study (Kim et al., 2020). They are listed in Table 1 . Since the radius of dislocation core, which was shown in previous study (Kim et al., 2020), was approximately $2 b$, we used $\sigma_{D}=\sigma_{G} b=2 b$ in this study. Then, we numerically calculated $f$ and $\left|F_{\text {drag }} / F_{P K}\right|\left(=C_{1}^{\text {dis }}\right.$ ) by solving Eq. (47) and (77), respectively. Here, we assumed that the fast-moving dislocation accompanied oscillation characterized by an anti-symmetric mode because it predominantly determines the drag force for fast-moving dislocation as we derived in Section 4.2. Finally, $C_{2}^{\text {dis }}$ could be obtained by the relation $C_{2}^{\text {dis }}=\left(\pi^{2} f^{2} / 2\right) C_{1}^{\text {dis }}$.

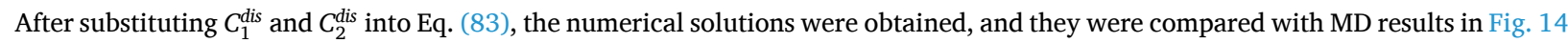
as well as Eq. (84) that were derived based on continuum theory (Dudorov and Mayer, 2011).

$$
v=\frac{C_{t} \varsigma}{6 \sqrt{6}}\left(\frac{(108 \varsigma+12 \sqrt{3} \sqrt{4+27 \varsigma})^{2 / 3}-12}{\varsigma(108 \varsigma+12 \sqrt{3} \sqrt{4+27 \varsigma})^{1 / 3}}\right)^{3 / 2},
$$

where $\zeta=M_{0} b\left(\tau_{a p p}-\tau_{c}\right) / C_{t}$ and $C_{t}$ is shear wave velocity. The input parameters for Eq. (84) are listed in Table 1. In Fig. 14(a), the dominant oscillation mode of dislocation core atoms is described. According to Figs. 14(b)-(d), our solutions described the MD results with great accuracy especially in high-velocity regime. On the contrary, the continuum-based solutions had a limitation to describe the dislocation velocity. This is because the continuum-based model oversimplified the relativistic effect by using the Lorentz factor $\left(1-\left(v / C_{t}\right)^{2}\right)^{-1 / 2}$ without considering the dislocation core structures. In other words, according to the continuum model, the relativistic effect was determined solely by the ratio $v / C_{t}$. This implies that energy dissipation due to dislocation oscillation must be considered when the dislocation velocity is sufficiently large. Therefore, our approach enables to describe the dislocation velocity-stress curve as well as the dominant oscillation mode of dislocation. Considering that the dislocation velocity response is directly connected to plastic strain rate by the Orowan equation, our work contributes to advance the pre-existing plasticity models based on better understanding of dislocation physics.

\subsection{Application to meso- and macro-scale plasticity models}

To date, most of previous studies using mesoscale dislocation dynamics have assumed constant dislocation mobility even when the dislocation glides under the extreme conditions. However, since the dislocation mobility is not constant but generally decreases with increasing dislocation velocity due to the phonon drag, the dislocation mobility under the extreme conditions shows large deviation from it under low stress or temperature. In addition, since the relativistic effect also has influence on the phonon drag at the extreme conditions by changing core width and frequency of dislocation during its motion (Wang and Beyerlein, 2008; Kim et al., 2020), our work enables to formulate their overall influences on the dislocation mobility at atomic level. From Eq. (83), the dislocation mobility in the limit of high stress (or high strain rate) can be derived as a function of stress and temperature:

$$
M\left(\tau_{a p p}, T\right)=\frac{1}{b} \frac{d v\left(\tau_{a p p}, T\right)}{d \tau_{a p p}} \approx \frac{M_{0}\left(1-C_{1}^{d i s}(T)\right)}{3-2\left(\frac{v_{0}}{v}\right)\left(1-C_{1}^{d i s}(T)\right)},
$$

where $v_{0}=M_{0} \tau_{a p p} b$. Eq. (85) explains the temperature-dependent dislocation mobility since $C_{1}^{\text {dis }}$ is a function of dislocation frequency and core width that are sensitive to temperature.

Furthermore, our model can be also extended to macroscale plasticity by relating flow stress with plastic strain rate through the dislocation mobility. According to Hunter and Preston (2015), the flow stress-strain rate relationship is derived based on mean-first-passage-time theory as:

$$
\dot{\varepsilon}=\frac{\varsigma\left(\tau_{e f f}\right) \rho_{m} b}{\sqrt{\rho_{i}} t_{r}^{e f f}\left(\tau_{e f f}\right)+B\left(v, \tau_{e f f}, T\right) / \tau_{e f f} b},
$$

where $\rho_{m}$ and $\rho_{i}$ are densities of mobile and immobile dislocation, respectively, $\tau_{\text {eff }}$ is effective stress acting on a dislocation, $t_{r}^{e f f}$ is effective mean remobilization time, $B=M^{-1}$ is the drag coefficient, and 
Table 1

Input parameters to compute Eqs. (83) and (84). These were obtained by MD simulations of an edge dislocation in 2D triangular lattices (Kim et al., 2020). The atomic interactions were described by Lennard-Jones (LJ) potential with arbitrary controlling the LJ parameter $\varepsilon$, which represents the depth of the potential well.

\begin{tabular}{lllllrr}
\hline Model & $\varepsilon[\mathrm{eV}]$ & $b[\AA]$ & $G[\mathrm{GPa}]$ & $M_{0}\left[\mathrm{~Pa}^{-1} \mathrm{~s}^{-1}\right]$ & $\omega_{L}\left[\times 10^{13} \mathrm{~s}^{-1}\right]$ & $\tau_{t}[\mathrm{~km} / \mathrm{s}]$ \\
\hline 1 & 0.3532 & 2.545 & 106.9 & 401 & 19.28 & 2.0 \\
2 & 0.7064 & & 213.8 & 275 & 2.09 & 2.95 \\
3 & 2.1192 & & 641.4 & 149 & 47.22 & 10 \\
\hline
\end{tabular}

(a)

(c)
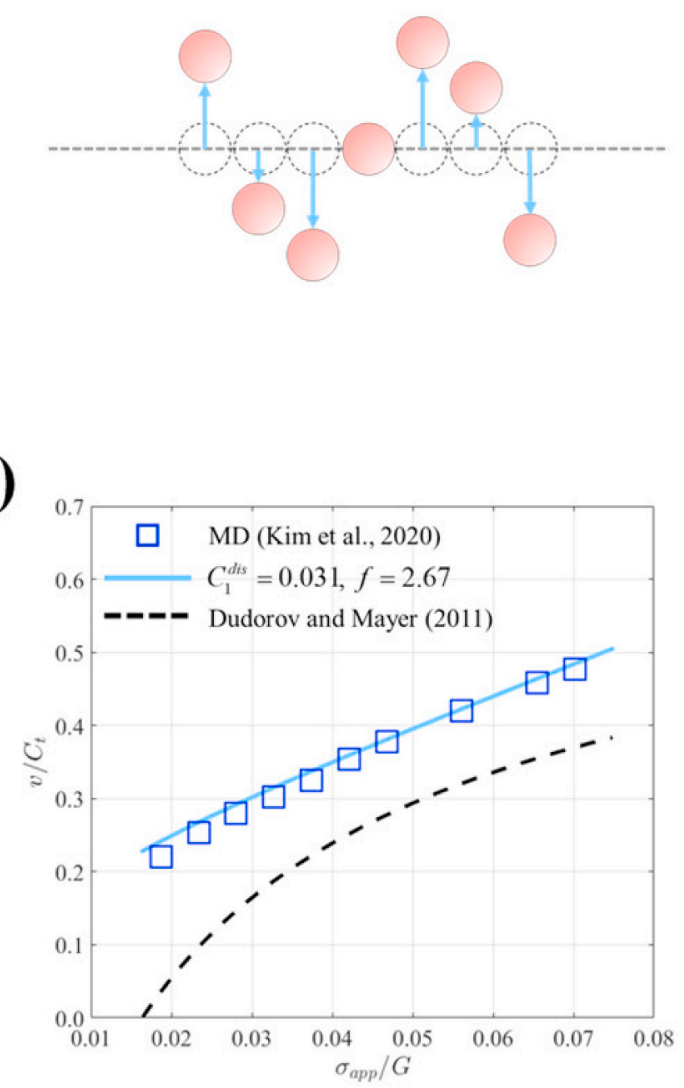

(b)

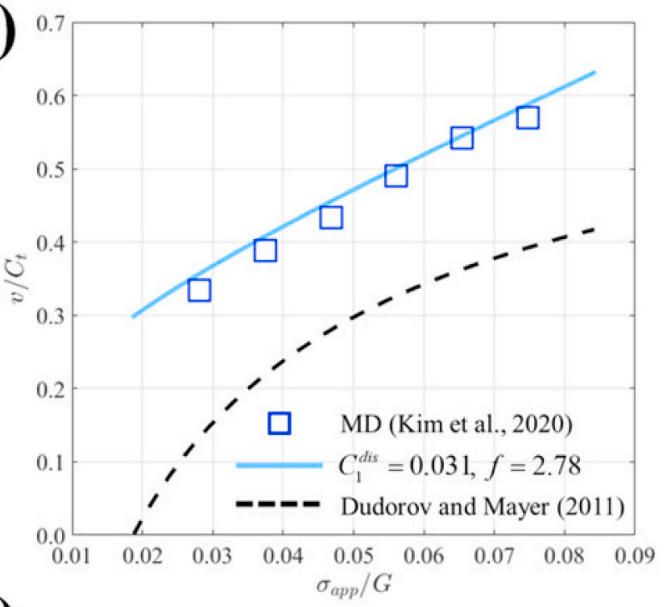

(d)

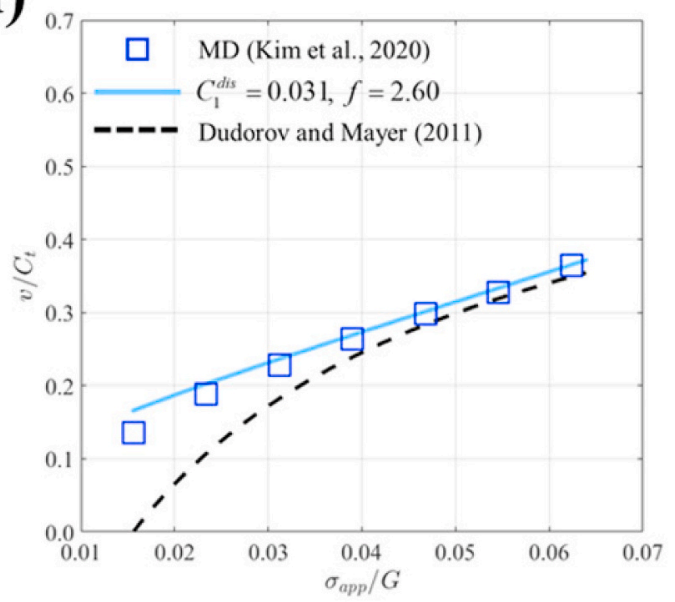

Fig. 14. (a) A dominant anti-symmetric oscillation mode of dislocation core atoms. Dashed circles are atomic positions before loading. Blue arrows represent atomic displacements due to oscillation. (b) Normalized dislocation velocity-stress relations for model 1, (c) model 2, and (d) model 3 for an edge dislocation in LJ crystals. Each model has different LJ parameters, which are given by Table 1. In the figures from (b)-(d), MD results (square points), solutions of Eq. (83) for specified $C_{1}^{\text {dis }}$ and $f$ (solid lines), and continuum-based solutions, or Eq. (84), (dashed lines) are compared.

$$
\varsigma\left(\tau_{\text {eff }}\right)=\left[1-\frac{1}{4} \exp \left(-\log _{10}^{2}\left(\frac{t_{r}^{e f f}\left(\tau_{\text {eff }}\right)}{t_{T}\left(\tau_{\text {eff }}\right)}\right)\right)\right]^{-1} .
$$

Here, $t_{T}$ is the transit time required for a dislocation to glide to the nearest obstacles. By substituting Eq. (82) into Eq. (86) through Eq. (85), we can finally investigate the influence of phonon drags on macroscale stress-strain rate relationship. In fact, the similar approach was very recently done by Blaschke et al. (2020b) and they emphasized the phonon drag even at intermediate strain rates. By using our theory, we can go further and provide the way to connect atomic-scale dislocation properties with the macroscale plasticity in multiscale framework. 


\section{Conclusion}

Based on the discrete lattice dynamics theory, we analytically derived the configurational drag force for each eigenmode by adopting dislocation-phonon coordinates. The drag force was responsible for the path-dependency of the $J$ integral around a moving dislocation and it was generated by a localized oscillation of the dislocation. By assuming the dislocation core as an isolated defect having a finite core width, the oscillation was simply classified into symmetric and anti-symmetric modes. The symmetric modes only interacted with the self-stress field of the dislocation, whereas the anti-symmetric modes only interacted with the externally applied stress. Consequently, the number of allowable eigenmodes increased as the core width increased. Moreover, more eigenmodes became activated as the core became stiffer. In particular, the SA mode had a lower frequency than the FA mode, and its displacement distribution was more localized at the edge atoms of the dislocation core than in the FA mode. Consequently, the SA mode produced a larger drag force than the FA mode. In addition, there was an inverse relationship between the drag force and the dislocation core width. This tendency was also observed in the previous study (Kim et al., 2020). Furthermore, we quantitatively proved that fundamental understandings of configurational force on a dislocation can be applied to predict dislocation velocity-stress curve for fast-moving dislocation as well as is dominant oscillation mode. Given that our work revealed the relation between the dislocation mobility and oscillation mode at most fundamental level, out work will pave the way for developing multiscale plasticity models where dislocation cores are well considered.

As a future study, this work can improve discrete dislocation dynamics (DDD) simulation when phonon dragging becomes important to determine flow stress because this study dealt with the dislocation motion accompanying high-frequency oscillation. In general, this happens when the material is exposed to extreme conditions such as high temperature or high strain rate. Furthermore, our work also provides insight to understand transient dislocation motion that usually occurs when dislocation accelerates or decelerates. Since the transient motion induces non-local response in time, the dislocation core width changes over time. As a result, the resulting dislocation self-force is history-dependent and dislocation has frequency-dependent effective mass (Pellegrini, 2012). Since we proved that the drag force also depends on both frequency and core width as well as the self-force, our work might enable DDD to describe transient dislocation motion in complete form.

\section{Declaration of competing interest}

The authors declare that they have no known competing financial interests or personal relationships that could have appeared to influence the work reported in this paper.

\section{Acknowledgments}

We gratefully acknowledge the support from the Mid-Career Researcher Support Program (Grant No. 2019R1A2C2011312) of the National Research Foundation of Korea and from the Meta-Structure Based Seismic Shielding Research Fund (Project No. 1.200043.01) of UNIST. We also acknowledge with gratitude the supercomputing resources of the UNIST Supercomputing Center.

\section{Appendix A. Lagrangian}

If we define the stresses and displacements caused by $D$ and $S$ as $\sigma^{D}$ and $\sigma^{S}$ and $u^{D}$ and $u^{S}$, respectively, the interaction potential energy, $U^{D S}$, becomes

$$
U^{D S}=\int_{I+I I}\left(\sigma_{i j}^{D} u_{i, j}^{S}+\sigma_{i j}^{S} u_{i, j}^{D}\right) d^{3} x
$$

As an elastic displacement is not defined at a singularity, $u^{D}$ and $u^{S}$ are not defined at $D$ and $S$, respectively. Thus, Eq. (A.1) is modified into

$$
U^{D S}=\int_{I} \sigma_{i j}^{D} u_{i, j}^{S} d^{3} x+\int_{I I} \sigma_{i j}^{S} u_{i, j}^{D} d^{3} x
$$

Using an integration by parts on the second term on the right-hand side (RHS) of Eq. (A.2), we obtain Eq. (A.3).

$$
U^{D S}=\int_{\Sigma_{o}} \sigma_{i j}^{S} u_{i}^{D} d S_{j}-\int_{\Sigma_{i}} \sigma_{i j}^{S} u_{i}^{D} d S_{j}-\int_{I I} \sigma_{i j, j}^{S} u_{i}^{D} d^{3} x+\int_{I} \sigma_{i j}^{D} u_{i, j}^{S} d^{3} x .
$$

The first integral in Eq. (A.3) represents the energy supplied from the external mechanism to the system, and thus, it indicates a decrease in the potential energy of the external mechanism. Therefore, if we extend the body of the system to include the external mechanism, the first integral in Eq. (A.3) is cancelled out. According to Fig. 1(b), as the surface integral on $\Sigma_{i}$ in Eq. (A.3) crosses a cut surface, $C$, a displacement jump occurs as much as $b$, which is the Burgers vector of the dislocation loop. Moreover, the elastic selfdisplacement field of the dislocation whose length is $l, u^{D}$, has an order $O(\ln a)$; hence, the second term in Eq. (A.3) satisfies 


$$
\int_{\Sigma_{i}} \sigma_{i j}^{S} u_{i}^{D} d S_{j} \sim-b_{i} \int_{C} \sigma_{i j}^{S} d S_{j}+\int_{0}^{l} \sigma_{i j}^{S}(\ln a)(2 \pi a) d l=-b_{i} \int_{C} \sigma_{i j}^{S} d S_{j}+O(a \ln a) .
$$

Moreover, as $\sigma^{D} \sim 1 / r$ within region I, the last term on the RHS of Eq. (A.3) becomes

$$
\int_{I} \sigma_{i j}^{D} u_{i, j}^{S} d^{3} x \sim \int_{0}^{a} \int_{0}^{l} \frac{1}{r} u_{i, j}^{D}(2 \pi r) d l d r=O(a) .
$$

Thus, as $a \rightarrow 0$, Eq. (A.3) becomes

$$
U^{D S}=b_{i} \int_{C} \sigma_{i j}^{S} d S_{j}-\int_{I+I I} \sigma_{i j, j}^{S} u_{i}^{D} d^{3} x .
$$

If the material velocities caused by $D$ and $S$ are defined as $v^{D}$ and $v^{S}$, respectively, Eq. (A.6) is modified to Eq. (A.7) by the force equilibrium equation.

$$
U^{D S}=b_{i} \int_{C} \sigma_{i j}^{S} d S_{j}-\int_{I+I I} \rho u_{i}^{D} \dot{v}_{i}^{S} d^{3} x
$$

where $\rho$ is the density of the system. The kinetic energy caused by the interaction between $D$ and $S$ is expressed by

$$
T^{D S}=\int_{I} \rho v_{i}^{D} v_{i}^{S} d^{3} x+\int_{I I} \rho v_{i}^{D} v_{i}^{S} d^{3} x .
$$

Within region I, as $u^{D} \sim \ln r$,

$$
T^{D S} \sim \int_{I I} \rho v_{i}^{D} v_{i}^{S} d^{3} x+\int_{0}^{l} \int_{0}^{a} \rho \frac{1}{r}(2 \pi r) d r d l=\int_{I I} \rho v_{i}^{D} v_{i}^{S} d^{3} x+O(a) .
$$

Here, notice that $v_{i}^{D}$ is well defined in region II because $D$ lies wholly in region I. From Eq. (A.7) and Eq. (A.9) with $a \rightarrow 0, L^{D S}$ is derived as

$$
\begin{gathered}
L^{D S}=T^{D S}-U^{D S} \\
=-b_{i} \int_{C} \sigma_{i j}^{S} d S_{j}+\int_{I+I I} \rho\left(u_{i}^{D} \dot{v}_{i}^{S}+\dot{u}_{i}^{D} v_{i}^{S}\right) d^{3} x \\
=-b_{i} \int_{C} \sigma_{i j}^{S} d S_{j}+\int_{I+I I} \rho \frac{d}{d t}\left(u_{i}^{D} v_{i}^{S}\right) d^{3} x .
\end{gathered}
$$

\section{Appendix B. Variation of the Lagrangian}

Assume that the dislocation loop, $D$, moves by an infinitesimal distance, $\delta \xi$, as shown in Fig. 2 . Then, a variation of the Lagrangian is given by

$$
\begin{gathered}
\delta L^{D S}=-\delta\left(b_{i} \int_{C} \sigma_{i j}^{S} d S_{j}\right)+\delta\left(\int_{I+I I} \rho \frac{d}{d t}\left(u_{i}^{D} v_{i}^{S}\right) d^{3} x\right) \\
=-b_{i} \int_{\delta V} \sigma_{i j, j}^{S} d^{3} x+b_{i} \oint \sigma_{i j}^{S} \varepsilon_{j k l} d s_{k} \delta \xi_{l}+\delta \int_{I+I I} \rho \frac{d}{d t}\left(u_{i}^{D} v_{i}^{S}\right) d^{3} x
\end{gathered}
$$

where $\delta V$ is the infinitesimal volume change due to the dislocation motion, $s_{k}$ is the unit vector along the dislocation line, and $\varepsilon_{j k l}$ is the permutation tensor. According to the force equilibrium equation without body force, $\sigma_{i j . j}^{S}=\rho \dot{v}_{i}^{S}$ is satisfied; hence, the first term on the RHS of Eq. (B.1) becomes

$$
b_{i} \int_{\delta V} \sigma_{i j, j}^{S} d^{3} x=\rho b_{i} \int_{\delta V} \dot{v}_{i}^{S} d^{3} x .
$$

As only the dislocation, $D$, moves, but not $S$, the variation of the last term in Eq. (B.1) is applied only to $u^{D}$ with the volume change, $\delta V$. Thus, the last term in Eq. (B.1) becomes 


$$
\delta \int_{I+I I} \rho \frac{d}{d t}\left(u_{i}^{D} v_{i}^{S}\right) d^{3} x=\int_{I+I I} \rho \frac{d}{d t}\left(\delta u_{i}^{D} v_{i}^{S}\right) d^{3} x+\int_{\delta V} \rho \frac{d}{d t}\left(u_{i}^{D} v_{i}^{S}\right) d^{3} x
$$

As $u_{i}^{D}=b_{i}$ is satisfied within $\delta V$, Eq. (B.3) becomes

$$
\delta \int_{I+I I} \rho \frac{d}{d t}\left(u_{i}^{D} v_{i}^{S}\right) d^{3} x=\int_{I+I I} \rho \frac{d}{d t}\left(\delta u_{i}^{D} v_{i}^{S}\right) d^{3} x+\rho b_{i} \int_{\delta V} \dot{v}_{i}^{S} d^{3} x
$$

Thus, by substituting Eq. (B.2) and Eq. (B.4) into Eq. (B.1), Eq. (B.5) is derived.

$$
\delta L^{D S}=b_{i} \oint \sigma_{i j}^{S} \varepsilon_{j k l} d s_{k} \delta \xi_{l}+\int_{I+I I} \rho \frac{d}{d t}\left(\delta u_{i}^{D} v_{i}^{S}\right) d^{3} x=\delta L_{1}^{D S}+\delta L_{2}^{D S} .
$$

If we define the first and second integrals in Eq. (B.5) as $\delta L_{1}^{D S}$ and $\delta L_{2}^{D S}$, respectively, the force acting on $D$, to which $\delta L_{1}^{D S}$ contributes, is derived as Eq. (B.6) according to Eq. (2).

$$
F_{\xi}^{1}=b_{i} \sigma_{i j}^{S} \varepsilon_{j k l} t_{k}
$$

where $t_{k}$ is the unit dislocation line vector. Furthermore, $\delta L_{2}^{D S}$ in Eq. (B.5) can be modified as

$$
\delta L_{2}^{D S}=\int_{I} \rho \frac{d}{d t}\left(\delta u_{i}^{D} v_{i}^{S}\right) d^{3} x+\int_{I I} \rho \frac{d}{d t}\left(\delta u_{i}^{D} v_{i}^{S}\right) d^{3} x=\int_{I} \rho \frac{d}{d t}\left(\delta u_{i}^{D} v_{i}^{S}\right) d^{3} x+\frac{d}{d t} \int_{I I} \rho \delta u_{i}^{D} v_{i}^{S} d^{3} x-\int_{I I} \rho \delta u_{i}^{D} v_{i}^{S} \frac{d}{d t}\left(d^{3} x\right)
$$

As $\xi$ is defined as the position of the dislocation relative to a stationary observer, or a point in region II, $d\left(d^{3} x\right) / d t=-\dot{\xi}_{j} d S_{j}$ is satisfied in region II. As $\delta \xi$ is zero at $t=t_{1}$ and $t=t_{2}$, the $\frac{d}{d t} \int_{I I} d^{3} x$ term does not contribute to the force acting on the dislocation. Thus,

$$
\delta L_{2}^{D S}=\int_{I} \rho \frac{d}{d t}\left(\delta u_{i}^{D} v_{i}^{S}\right) d^{3} x+\int_{\Sigma_{i}} \rho \delta u_{i}^{D} v_{i}^{S} \dot{\xi}_{j} d S_{j}=\int_{\Sigma_{i}} \rho \delta u_{i}^{D} v_{i}^{S} \dot{\xi}_{j} d S_{j}+\int_{I} \rho v_{i}^{S} \frac{d}{d t}\left(\delta u_{i}^{D}\right) d^{3} x+\int_{I} \rho \dot{v}_{i}^{S} \delta u_{i}^{D} d^{3} x
$$

Here, as $u_{i}^{D} \sim \ln r$ in region $\mathrm{I}, \delta u_{i}^{D} \sim 1 / r$ so that

$$
\int_{I} \rho \dot{v}_{i}^{S} \delta u_{i}^{D} d^{3} x \sim \int_{0}^{l} \int_{0}^{a} \rho \dot{v}_{i}^{S} \frac{1}{r}(2 \pi r) d r d l=O(a) .
$$

As $a \rightarrow 0, \delta L_{2}^{D S}$ results in

$$
\delta L_{2}^{D S}=\int_{\Sigma_{i}} \rho \delta u_{i}^{D} v_{i}^{S} \dot{\xi}_{j} d S_{j}+\int_{I} \rho v_{i}^{S} \frac{d}{d t}\left(\delta u_{i}^{D}\right) d^{3} x
$$

\section{Appendix C. The kinetic component of configurational force}

If Eq. (16-1) is differentiated by time,

$$
\dot{u}_{n}=\left[\frac{d w_{n}}{d \xi}-\frac{d \lambda_{n}^{(1)}}{d \xi} \sum_{m} \lambda_{m}^{(1)} v_{m}-\lambda_{n}^{(1)} \sum_{m} \frac{d \lambda_{m}^{(1)}}{d \xi} v_{m}\right] \dot{\xi}+\dot{v}_{n}-\lambda_{n}^{(1)} \sum_{m} \lambda_{m}^{(1)} \dot{v}_{m}
$$

is derived. From Eq. (C.1), we obtain Eq. (C.2)

$$
\sum_{n} \dot{u}_{n}^{2}=A \dot{\xi}^{2}+\sum_{n} \dot{v}_{n}^{2}+2 \sum_{n} p_{n} \dot{v}_{n} \dot{\xi}
$$

where

$$
\begin{gathered}
p_{n}=\frac{d w_{n}}{d \xi}-\frac{d \lambda_{n}^{(1)}}{d \xi} \sum_{m} \lambda_{m}^{(1)} v_{m}-\lambda_{n}^{(1)} \\
A=\sum_{n}\left(\frac{d w_{n}}{d \xi}\right)^{2}+\sum_{n}\left(\frac{d w_{n}}{d \xi}\right)^{2}\left(\sum_{m} \lambda_{m}^{(1)} v_{m}\right)^{2}+\left(\sum_{n} \frac{d \lambda_{n}^{(1)}}{d \xi} v_{n}\right)^{2}-2\left(\sum_{n} \frac{d w_{n}}{d \xi} \frac{d \lambda_{n}^{(1)}}{d \xi}\right)\left(\sum_{m} \lambda_{m}^{(1)} v_{m}\right) \\
-2\left(\sum_{n} \lambda_{n}^{(1)} \frac{d w_{n}}{d \xi}\right)\left(\sum_{m} \frac{d \lambda_{m}^{(1)}}{d \xi} v_{m}\right)+O\left(v^{2}\right),
\end{gathered}
$$


Since $\lambda_{n}^{(1)}=d w_{n} / d \xi$ and $\sum_{n}\left(\lambda_{n}^{(1)}\right)^{2}=1$ are satisfied by Eqs. (9) and (13), respectively, Eq. (C.3) becomes

$$
p_{n}=-\frac{d \lambda_{n}^{(1)}}{d \xi} \sum_{m} \lambda_{m}^{(1)} v_{m}
$$

If we express Eq. (C.4) by using Eq. (C.5) and neglect $O\left(v^{2}\right)$ term, Eq. (C.4) is rewritten as

$$
A=\sum_{n} p_{n}^{2}+\left(1-\sum_{n} \frac{d \lambda_{n}^{(1)}}{d \xi} v_{n}\right)^{2}-2\left(\sum_{n} \lambda_{n}^{(1)} \frac{d \lambda_{n}^{(1)}}{d \xi}\right)\left(\sum_{m} \lambda_{m}^{(1)} v_{m}\right)
$$

Since $\lambda_{n}^{(1)}\left(d \lambda_{n}^{(1)} / d \xi\right)$ is odd function of $x$, Eq. (C.6) is reduced to Eq. (C.7).

$$
A=\sum_{n} p_{n}^{2}+B^{2}
$$

where $B=1-\sum_{n}\left(d \lambda_{n}^{(1)} / d \xi\right) v_{n}$. As a result, Eq. (C.2) becomes

$$
\sum_{n} \dot{u}_{n}^{2}=B^{2} \dot{\xi}^{2}+\sum_{n}\left(p_{n} \dot{\xi}+\dot{v}_{n}\right)^{2}
$$

Therefore, the system's kinetic energy becomes

$$
T^{s y s}=\frac{1}{2} m B^{2} \dot{\xi}^{2}+\frac{1}{2} m \sum_{n}\left(p_{n} \dot{\xi}+\dot{v}_{n}\right)^{2}
$$

Here, if we assume that the phonon wavelength is much larger than the core width (i.e., $\left.\sum_{n}\left(d \lambda_{n}^{(1)} / d \xi\right) v_{n} \approx(v / b) \int\left(d \lambda^{(1)} / d \xi\right) d x=0\right)$ and $\dot{\xi} \gg \dot{v}_{n}$ (Ninomiya, 1972; Kim et al., 2016), then Eq. (C.9) is rewritten as

$$
T^{s y s}=\frac{1}{2} m\left(1+\sum_{n} p_{n}^{2}\right) \dot{\xi}^{2}
$$

As a result, Eq. (C.11) is derived.

$$
\begin{gathered}
\frac{\partial T^{s y s}}{\partial \xi}=m \dot{\xi}^{2} \sum_{n} p_{n} \frac{\partial p_{n}}{\partial \xi} \\
=m \dot{\xi}^{2}\left(\sum_{n} \frac{d \lambda_{n}^{(1)}}{d \xi} \frac{d^{2} \lambda_{n}^{(1)}}{d \xi^{2}}\right)\left(\sum_{m} \lambda_{m}^{(1)} v_{m}\right)^{2}+m \dot{\xi}^{2}\left(\sum_{n} \frac{d \lambda_{n}^{(1)}}{d \xi}\right)^{2}\left(\sum_{m} \lambda_{m}^{(1)} v_{m}\right)\left(\sum_{l} \frac{d \lambda_{l}^{(1)}}{d \xi} v_{l}\right) .
\end{gathered}
$$

From the definition of $\lambda_{n}^{(1)}(\xi)$ given by Eq. (22),

$$
\frac{d \lambda_{n}^{(1)}}{d \xi}=\frac{b(r-\xi)}{\sigma_{D}^{3} \sqrt{2 \pi}} \exp \left(-\frac{(r-\xi)^{2}}{2 \sigma_{D}^{2}}\right)
$$

and

$$
\frac{d^{2} \lambda_{n}^{(1)}}{d \xi^{2}}=\frac{b}{\sigma_{D}^{3} \sqrt{2 \pi}}\left(-1+\frac{(r-\xi)^{2}}{\sigma_{D}^{2}}\right) \exp \left(-\frac{(r-\xi)^{2}}{2 \sigma_{D}^{2}}\right)
$$

are obtained. Since we assume that the dislocation is at the origin, and hence, $\xi=0$, the first parenthesis of Eq. (C.11) becomes:

$$
\left.\sum_{n} \frac{d \lambda_{n}^{(1)}}{d \xi} \frac{d^{2} \lambda_{n}^{(1)}}{d \xi^{2}} \approx \frac{1}{b} \int_{-\infty}^{\infty}\left(\frac{d \lambda_{n}^{(1)}}{d \xi} \frac{d^{2} \lambda_{n}^{(1)}}{d \xi^{2}}\right)\right|_{\xi=0} d r=\frac{b}{2 \pi \sigma_{D}^{6}} \int_{-\infty}^{\infty} r\left(-1+\frac{r^{2}}{\sigma_{D}^{2}}\right) e^{-\frac{r^{2}}{\sigma_{D}^{2}}} d r
$$

Since the integrand of Eq. (C.14) is odd function of $r, \sum_{n} \frac{d_{n}^{(1)}}{d \xi} \frac{d^{2} \lambda_{n}^{(1)}}{d \xi^{2}}$ becomes zero. In the same way,

$$
\left.\sum_{l} \frac{d \lambda_{l}^{(1)}}{d \xi} v_{l} \approx \frac{v}{b} \int_{-\infty}^{\infty} \frac{d \lambda_{l}^{(1)}}{d \xi}\right|_{\xi=0} d r=\frac{v}{\sigma_{D}^{3} \sqrt{2 \pi}} \int_{-\infty}^{\infty} r e^{-\frac{r^{2}}{2 \sigma_{D}^{2}}} d r=0
$$

is satisfied under the long wavelength assumption. By substituting Eq. (C.14) and (C.15) into Eq. (C.11), $\partial T^{\text {sys }} / \partial \xi=0$ is finally derived. 


\section{References}

Agiasofitou, E., Lazar, M., 2017. Micromechanics of dislocations in solids: J-, M-, and L-integrals and their fundamental relations. Int. J. Eng. Sci. 114, 16-40. Al'Shitz, V.A., Indenbom, V.L., 1975. Dynamic dragging of dislocations. Sov. Phys. Usp. 18, 1-20.

Amodeo, J., Dancette, S., Delannay, L., 2016. Atomistically-informed crystal plasticity in MgO polycrystals under pressure. Int. J. Plast. 82, $177-191$.

Amrit, J., Ramiere, A., Volz, S., 2018. Role of fluttering dislocations in the thermal interface resistance between a silicon crystal and plastic solid ${ }^{4}$ He. Phys. Rev. B 97 , 014308.

Arvanitakis, A.I., Chronaiou, E.I., Kalpakides, V.K., 2012. On the dynamics of moving singularities in solids under the use of the level-set method and the configurational force concept. Compos. B Eng. 43, 2628-2632.

Ballarini, R., Royer-Carfagni, G., 2016. A Newtonian interpretation of configurational forces on dislocations and cracks. J. Mech. Phys. Solid. 95, 602-620.

Baxevanakis, K.P., Giannakopoulos, A.E., 2015. Finite element analysis of discrete edge dislocations: configurational forces and conserved integrals. Int. J. Solid Struct. 62, 52-65.

Blaschke, D.N., 2019a. Velocity dependent dislocation drag from phonon wind and crystal geometry. J. Phys. Chem. Solid. 124, 24-35.

Blaschke, D.N., 2019b. Properties of dislocation drag from phonon wind at ambient conditions. Materials $12,948$.

Blaschke, D.N., Mottola, E., Preston, D.L., 2020a. Dislocation drag from phonon wind in an isotropic crystal at large velocities. Phil. Mag. 100, 571-600.

Blaschke, D.N., Hunter, A., Preston, D.L., 2020b. Analytic model of the remobilization of pinned glide dislocations: including dislocation drag from phonon wind. Int. J. Plast. https://doi.org/10.1016/j.ijplas.2020.102750 (article (in press).

Budiansky, B., Rice, J.R., 1973. Conservation laws and energy-release rates. J. Appl. Mech. 40, 201-203.

Chen, X., Xiong, L., McDowell, D., Chen, Y., 2017. Effects of phonons on mobility of dislocations and dislocation arrays. Scripta Mater. $137,22-26$.

Cui, Y., Po, G., Pellegrini, Y.-P., Lazar, M., Ghoniem, N., 2019. Computational 3-dimensional dislocation elastodynamics. J. Mech. Phys. Solid. 126, 20-51. https:// doi.org/10.1016/j.jmps.2019.02.008.

Dudorov, A.E., Mayer, A.E., 2011. The equations of the dynamics and kinetics of dislocations at high strain rate plastic deformation. CSU Bull. Phys. 39 (39), 48-56. Eshelby, J.D., 1951. The force on an elastic singularity. Phil. Trans. Roy. Soc. A 244, 87-112.

Eshelby, J.D., 1956. The continuum theory of lattice defects. Solid State Phys. 3, 79-144.

Guo, Y., Li, Q., 2017. Material configurational forces applied to mixed mode crack propagation. Theor. Appl. Fract. Mech. 89, $147-157$.

Gurrutxaga-Lerma, B., Balint, D.S., Dini, D., Eakins, D.E., Sutton, A.P., 2015. Attenuation of the dynamic yield point of shocked aluminum using elastodynamic simulations of dislocation dynamics. Phys. Rev. Lett. 114, 174301.

Gurrutxaga-Lerma, B., 2016. The role of the mobility law of dislocations in the plastic response of shock loaded pure metals. Model. Simulat. Mater. Sci. Eng. 24, 065006.

Hossain, M.Z., Hsueh, C.-J., Bourdin, B., Bhattacharya, K., 2014. Effective toughness of heterogeneous media. J. Mech. Phys. Solid. 71, 15-32.

Hunter, A., Preston, D.L., 2015. Analytic model of the remobilization of pinned glide dislocations from quasi-static to high strain rates. Int. J. Plast. 70, 1-29.

Jamond, O., Gatti, R., Roos, A., Devincre, B., 2016. Consistent formulation for the discrete-continuous model: improving complex dislocation dynamics simulations. Int. J. Plast. 80, 19-37.

Kaczmarczyk, Ł., Nezhad, M.M., Pearce, C., 2014. Three-dimensional brittle fracture: configurational-force-driven crack propagation. Int. J. Numer. Methods Eng. 97, $531-550$.

Kim, S., Ho, D.T., Kang, K., Kim, S.Y., 2016. Phonon scattering during dislocation motion inducing stress-drop in cubic metals. Acta Mater. 115 , $143-154$.

Kim, S., Kim, H., Kang, K., Kim, S.Y., 2020. Relativistic effect inducing drag on fast-moving dislocation in discrete system. Int. J. Plast. $126,102629$.

Kosevich, A.M., 1965. Dynamical theory of dislocations. Sov. Phys. Usp. 7, 837-854.

Kresse, O., Truskinovsky, L., 2003. Mobility of lattice defects: discrete and continuum approaches. J. Mech. Phys. Solid. 51, $1305-1332$.

Kuhn, C., Müller, R., 2016. A discussion of fracture mechanisms in heterogeneous materials by means of configurational forces in a phase field fracture model. Comput. Methods Appl. Mech. Eng. 312, 95-116.

Lee, D.W., Kim, H., Strachan, A., Koslowski, M., 2011. Effect of core energy on mobility in a continuum dislocation model. Phys. Rev. B 83, 104101.

Leung, H.S., Leung, P.S.S., Cheng, B., Ngan, A.H.W., 2015. A new dislocation-density-function dynamics scheme for computational crystal plasticity by explicit consideration of dislocation elastic interactions. Int. J. Plast. 67, 1-25.

Lifshitz, I.M., Kosevich, A.M., 1966. The dynamics of a crystal lattice with defects. Rep. Prog. Phys. 29, $217-254$.

Liu, G., Wang, J., Chen, K., Shen, Y., 2016. Peierls stress in face-centered-cubic metals predicted from an improved semi-discrete variation Peierls-Nabarro model. Scripta Mater. 120, 94-67.

Lothe, J., 1961. Lorentz force on screw dislocations and related problems. Phys. Rev. 122, 78-82.

Lubarda, V.A., Markenscoff, X., 2007. Configurational force on a lattice dislocation and the Peierls stress. Arch. Appl. Mech. 77, $147-154$.

Lubarda, V.A., 2015. Determination of interaction forces between parallel dislocations by the valuation of $J$ integrals of plane elasticity. Continuum Mech. Therm. 28, $391-405$

Luscher, D.J., Mayeur, J.R., Mourad, H.M., Hunter, A., Kenamond, M.A., 2016. Coupling continuum dislocation transport with crystal plasticity for application to shock loading conditions. Int. J. Plast. 76, 111-129.

Luscher, D.J., Buechler, M.A., Walters, D.J., Bolme, C.A., Ramos, K.J., 2018. On computing the evolution of temperature for materials under dynamic loading. Int. J. Plast. 111, 188-210.

Montroll, E.W., Potts, R.B., 1955. Effect of defects on lattice vibrations. Phys. Rev. 100, 525-543.

Ni, L., Markenscoff, X., 2008. The self-force and effective mass of a generally accelerating dislocation I: screw dislocation. J. Mech. Phys. Solid. 56, $1348-1379$.

Ninomiya, T., 1972. A theory of dislocation motion in a crystal. I. General and application to one-dimensional lattice. J. Phys. Soc. Jpn. 33, 921-928.

Ninomiya, T., 1974. Frictional force acting on a dislocation -Fluttering mechanism-. J. Phys. Soc. Jpn. 36, 399-405.

Noether, E., 1971. Invariant variation problems. Transport Theor. Stat. Phys. 1, 186-207.

Özenç, K., Chinaryan, G., Kaliske, M., 2016. A configurational force approach to model the branching phenomenon in dynamic brittle fracture. Eng. Fract. Mech. 157, $26-42$.

Pellegrini, Y.-P., 2012. Screw and edge dislocations with time-dependent core width: from dynamical core equations to an equation of motion. J. Mech. Phys. Solid. 60, 227-249.

Peng, S., Wei, Y., Jin, Z., Yang, W., 2019. Supersonic screw dislocations gliding at the shear wave speed. Phys. Rev. Lett. 122 , 045501.

Rice, J.R., 1968. A path-independent integral and the approximate analysis of strain concentrations by notes and cracks. J. Appl. Mech. 35, 379-388.

Seo, Y., Jung, G.-Y., Kim, I.-H., Pak, Y.E., 2018. Configurational forces on elastic line singularities. J. Appl. Mech. 85, 034501.

Shehadeh, M.A., Zbib, H.M., 2016. On the homogeneous nucleation and propagation of dislocations under shock compression. Phil. Mag. 96, $2752-2778$.

Slepyan, L.I., 2001. Feeding and dissipative waves in fracture and phase transition. I. Some 1D structures and a square-cell lattice. J. Mech. Phys. Solid. 49, 469-511.

Song, Q., Li, Z., Zhu, Y., Huang, M., 2018. On the interaction of solute atoms with circular inhomogeneity and edge dislocation. Int. J. Plast. 111, 266-287.

Stroh, A.N., 1962. Force on a moving dislocation. Phys. Rev. 128, 55-61.

Sun, Y., Zhou, Y., Han, J., Liu, W., Nan, C., Lin, Y., Hu, M., Xu, B., 2019. Strong phonon localization in PbTe with dislocations and large deviation to Matthiessen's rule. npj Comput. Mater. 5, 97.

Swinburne, T.D., Dudarev, S.L., Fitzgerald, S.P., Gilbert, M.R., Sutton, A.P., 2013. Theory and simulation of the diffusion of kinks on dislocations in bcc metals. Phys, Rev. B 87, 064108.

Wang, L., Abeyaratne, R., 2018. A one-dimensional peridynamic model of defect propagation and its relation to certain other continuum models. J. Mech. Phys. Solid. $116,334-349$. 
Wang, Z.Q., Beyerlein, I.J., 2008. Stress orientation and relativistic effects on the separation of moving screw dislocations. Phys. Rev. B 77, 184112.

Yuan, Z., Li, Q., 2019. A configurational force based anisotropic damage model for original isotropic materials. Eng. Fract. Mech. 215, 49-64.

Zhang, Y., Ngan, A.H.W., 2018. Dislocation-density dynamics for modeling the cores and Peierls stress of curved dislocations. Int. J. Plast. 104, 1-22. 